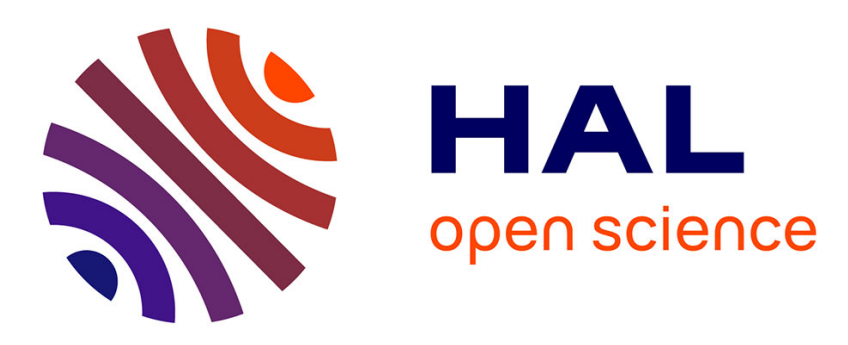

\title{
On strong regular stabilizability for linear neutral type systems
}

\author{
Rabah Rabah, Grigory M. Sklyar, Aleksandr V. Rezounenko
}

\section{To cite this version:}

Rabah Rabah, Grigory M. Sklyar, Aleksandr V. Rezounenko. On strong regular stabilizability for linear neutral type systems. Journal of Differential Equations, 2008, 245 (3), pp.569-593. 10.1016/j.jde.2008.02.041 . hal-00580044

\section{HAL Id: hal-00580044 https://hal.science/hal-00580044}

Submitted on 25 Mar 2011

HAL is a multi-disciplinary open access archive for the deposit and dissemination of scientific research documents, whether they are published or not. The documents may come from teaching and research institutions in France or abroad, or from public or private research centers.
L'archive ouverte pluridisciplinaire HAL, est destinée au dépôt et à la diffusion de documents scientifiques de niveau recherche, publiés ou non, émanant des établissements d'enseignement et de recherche français ou étrangers, des laboratoires publics ou privés. 


\title{
On strong regular stabilizability for linear neutral type systems
}

\author{
R. Rabah ${ }^{\text {a }}$ G. M. Sklyar ${ }^{b}$ A. V. Rezounenko ${ }^{c}$ \\ ${ }^{\mathrm{a}}$ IRCCyN UMR 6597, École des Mines de Nantes, \\ 4 rue Alfred Kastler BP 20722 44307 Nantes Cedex 3, France \\ Phone: (33) 251858311, Fax: (33) 251858349. Email: rabah@emn.fr \\ ${ }^{\mathrm{b}}$ Institute of Mathematics, University of Szczecin, \\ Wielkopolska 15, 70451 Szczecin, Poland.Email: sklar@univ.szczecin.pl \\ ${ }^{\mathrm{c}}$ Department of Mechanics and Mathematics, Kharkov National University, \\ 4 Svobody sqr., Kharkov,61077, Ukraine.Email: rezounenko@univer.kharkov.ua
}

\begin{abstract}
The problem of strong stabilizability of linear systems of neutral type is investigated. We are interested in the case when the system has an infinite sequence of eigenvalues with vanishing real parts. This is the case when the main part of the neutral equation is not assumed to be stable in the classical sense. We discuss the notion of regular strong stabilizability and present an approach to stabilize the system by regular linear controls. The method covers the case of multivariable control and is essentially based on the idea of infinite-dimensional pole assignment proposed in [G.Sklyar, A.Rezounenko, A theorem on the strong asymptotic stability and determination of stabilizing controls. C. R. Acad. Sci. Paris Ser. I Math. 333(2001), No. 8, 807812]. Our approach is based on the recent results on the Riesz basis of invariant finite-dimensional subspaces and strong stability for neutral type systems presented in [R. Rabah, G.M. Sklyar, A.V. Rezounenko, Stability analysis of neutral type systems in Hilbert space. J. of Differential Equations, 214(2005), No. 2, 391-428].

Keywords. Neutral type systems, regular stabilizability, strong stabilizability, infinite dimensional systems.
\end{abstract}

Mathematical subject classification. 93D15, 93C23.

\section{Introduction and problem formulation}

The field of delay differential equations has a rich history and many important and deep results have been obtained so far (see the classical monographs $[1,4,6])$. Neutral type differential equations belong to this field but many methods and approaches used for delay equations can not be directly applied to 
this type of equations.

We consider the following neutral type system

$$
\dot{z}(t)=A_{-1} \dot{z}(t-1)+\int_{-1}^{0} A_{2}(\theta) \dot{z}(t+\theta) \mathrm{d} \theta+\int_{-1}^{0} A_{3}(\theta) z(t+\theta) \mathrm{d} \theta
$$

where $A_{-1}$ is constant $n \times n$-matrix, $\operatorname{det} A_{-1} \neq 0, A_{2}, A_{3}$ are $n \times n$-matrices whose elements belong to $L_{2}(-1,0)$.

In our previous work [18] we analyzed asymptotic stability conditions. One of the main point of the cited work is the fact that for (1) it may appear asymptotic non exponential stability (see also [2] for the behavior of solutions of a class of neutral type systems). We gave a detailed analysis of non exponential stability in terms of the spectral properties of the matrix $A_{-1}$. As a continuation of those results we consider in the present work the control system

$$
\dot{z}(t)=A_{-1} \dot{z}(t-1)+\int_{-1}^{0} A_{2}(\theta) \dot{z}(t+\theta) \mathrm{d} \theta+\int_{-1}^{0} A_{3}(\theta) z(t+\theta) \mathrm{d} \theta+B u,
$$

where $B$ is a $n \times p$-matrix, and study the property for this system of being asymptotic stable after a choice of a feedback control law. Namely, we say that the system (2) is asymptotically stabilizable if there exists a linear feedback control $u(t)=F\left(z_{t}(\cdot)\right)=F(z(t+\cdot))$ such that the system (2) becomes asymptotically stable.

It is obvious that for linear systems in finite dimensional spaces the linearity of the feedback implies that the control is bounded in every neighborhood of the origin. For infinite dimensional spaces the situation is much more complicated. The boundedness of the feedback law $u=F\left(z_{t}(\cdot)\right)$ depends on the topology of the state space.

Let us observe that the natural state space for the equation (1) is the space of absolutely continuous functions $z(t+\theta), \theta \in[-1,0]$, since in this case the left hand side is correctly defined. Furthermore, it is convenient for us to assume that $z(t+\cdot) \in H^{1}\left([-1,0], \mathbb{C}^{n}\right)$. Then it can be shown (cf. $\left.[9,15]\right)$ that if the control function $u$ is in $L_{2}$, then the system (2) has an unique solution in the space $H^{1}\left([-1,0], \mathbb{C}^{n}\right)$. Hence, this space is natural as a state space also for the system (2). When the asymptotic stabilizability is achieved by a feedback law which does not change the state space and is bounded with respect to the topology of the state space, then we call it regular asymptotic stabilizability. Under our assumption on the state space, namely $H^{1}\left([-1,0], \mathbb{C}^{n}\right)$, the natural 
linear feedback is

$$
F z(t+\cdot)=\int_{-1}^{0} F_{2}(\theta) \dot{z}(t+\theta) \mathrm{d} t+\int_{-1}^{0} F_{3}(\theta) z(t+\theta) \mathrm{d} t
$$

where $F_{2}(\cdot), F_{3}(\cdot) \in L_{2}\left(-1,0 ; \mathbb{C}^{n}\right)$.

Several authors (see for example $[7,10,11]$ and references therein) use feedback laws which for our system may take the form

$$
\sum_{i=1}^{k} F_{i} \dot{z}\left(t-h_{i}\right)+\int_{-1}^{0} F_{2}(\theta) \dot{z}(t+\theta) \mathrm{d} t+\int_{-1}^{0} F_{3}(\theta) z(t+\theta) \mathrm{d} t .
$$

This feedback law is not bounded in $H^{1}\left([-1,0], \mathbb{C}^{n}\right)$ and then stabilizability is not regular. Later we shall return to this issue in more detail.

To make our concept more precise we consider the operator model of the system used in [18] (see also [3]):

$$
\dot{x}=\mathcal{A} x+\mathcal{B} u, \quad x(t)=\left(\begin{array}{c}
y(t) \\
z_{t}(\cdot)
\end{array}\right),
$$

where $\mathcal{A}$ is the generator of a $C_{0}$-semigroup and is defined by

$$
\mathcal{A} x(t)=\mathcal{A}\left(\begin{array}{c}
y(t) \\
z_{t}(\cdot)
\end{array}\right)=\left(\begin{array}{c}
\int_{-1}^{0} A_{2}(\theta) \dot{z}_{t}(\theta) \mathrm{d} \theta+\int_{-1}^{0} A_{3}(\theta) z_{t}(\theta) \mathrm{d} \theta \\
\mathrm{d} z_{t}(\theta) / \mathrm{d} \theta
\end{array}\right),
$$

with the domain

$$
\mathcal{D}(\mathcal{A})=\left\{(y, z(\cdot)): z \in H^{1}\left([-1,0] ; \mathbb{C}^{n}\right), y=z(0)-A_{-1} z(-1)\right\} \subset M_{2},
$$

where $M_{2} \stackrel{\text { def }}{=} \mathbb{C}^{n} \times L_{2}\left(-1,0 ; \mathbb{C}^{n}\right)$. The operator $\mathcal{B}: \mathbb{C}^{p} \rightarrow M_{2}$ is defined by the $n \times p$-matrix $B$ as follows

$$
\mathcal{B} u \stackrel{\text { def }}{=}\left(\begin{array}{c}
B u \\
0
\end{array}\right)
$$

The relation between the solution of the delay system (2) and the system (5) is $z_{t}(\theta)=z(t+\theta), \theta \in[-1,0]$. This model was used in particular in [18] for the analysis of the stability of the system (1) and in [15] for the analysis of the controllability problems (see also [3,22]). 
From the operator point of view, the regular feedback law (3) means a perturbation of the infinitesimal generator $\mathcal{A}$ by the operator $\mathcal{B} \mathcal{F}$ which is relatively $\mathcal{A}$-bounded (cf. [8]) and verifies $\mathcal{D}(\mathcal{A})=\mathcal{D}(\mathcal{A}+\mathcal{B} \mathcal{F})$. Such a perturbation does not mean, in general, that $\mathcal{A}+\mathcal{B F}$ is the infinitesimal generator of a $C_{0^{-}}$ semigroup. However, in our case, this fact is verified directly $[18,15]$ since after the feedback we get also a neutral type system like $(1)$ with $\mathcal{D}(\mathcal{A})=\mathcal{D}(\mathcal{A}+\mathcal{B} \mathcal{F})$ (see below for more details).

From a physical point of view, $\mathcal{A}$-boundedness of the stabilizing feedback $\mathcal{F}$ means that the energy added by the feedback remains uniformly bounded in every neighborhood of 0 . Hence the problem of regular asymptotic stabilizability for the systems $(2),(5)$ is to find a linear relatively $\mathcal{A}$-bounded feedback $u=\mathcal{F} x$ such that the operator $\mathcal{A}+\mathcal{B F}$ generates a $C_{0}$-semigroup $\mathrm{e}^{(\mathcal{A}+\mathcal{B} \mathcal{F}) t}$ with $\mathcal{D}(\mathcal{A}+\mathcal{B F})=\mathcal{D}(\mathcal{A})$ and for which $\left\|\mathrm{e}^{(\mathcal{A}+\mathcal{B F}) t} x\right\| \rightarrow 0$, as $t \rightarrow \infty$ for all $x \in \mathcal{D}(\mathcal{A})$.

If we take a feedback law in the form (4) then it means that the operator $\mathcal{A}$ is perturbed by a linear feedback unbounded with respect to the graph norm of $\mathcal{A}$, moreover the domain of the perturbed operator $\mathcal{D}(\mathcal{A}+\mathcal{B \mathcal { F }})$ is not equal to $\mathcal{D}(\mathcal{A})$. In [7] similar neutral type systems are considered in $C\left([-1,0] ; \mathbb{C}^{n}\right)$. The state space in this case is a subspace of $C^{1}\left([-1,0] ; \mathbb{C}^{n}\right)$ which depends on the right hand side of the system. So for any choice of the feedback $u=F(z(\cdot))$ in the operator model of the system we have $\mathcal{D}(\mathcal{A}+\mathcal{B} \mathcal{F}) \neq \mathcal{D}(\mathcal{A})$ and then the state space for the closed loop system is not the same as for the original plant. For this system a feedback like (4), namely

$$
\sum_{i=1}^{k} F_{i} \dot{x}\left(t-h_{i}\right)+\int_{-1}^{0} \mathrm{~d} G(\theta) x(t+\theta),
$$

where $G$ is of bounded variation, is used to achieve the exponential stability. In this context we notice that the feedback law of the type (9) is not regular.

The problem of regular asymptotic stabilization formulated here is considered within the concept of strong stability for infinite dimensional systems (see for example [12] and references therein). This approach was developed in the last decades when it was pointed out that asymptotic stability (called strong stability) may occur even if the norm of the semigroup does not tend to zero. For the system (1) the strong stability was investigated in our previous works $[17,18]$. For a particular case of neutral type systems the problem of strong stabilizability (regular in the sense of Definition 2) was considered in $[13,14]$. We here use the term of regular stabilizability for the neutral type system (in parallel with "strong stabilizability") also by the reason that for those systems the words "strong stabilizability" was used in [7] in an essentially different sense. So using the terms regular stabilizability, we would like to 
avoid a confusion, and also characterize the feedback quality.

Let us precise that if the feedback $\mathcal{F}$ is regular then the sets $\sigma(\mathcal{A}) \cap\{\lambda:|\lambda|>$ $R\}$ and $\sigma(\mathcal{A}+\mathcal{B F}) \cap\{\lambda:|\lambda|>R\}$ are asymptotically close when $R \rightarrow \infty$, cf. [18] and Theorem 4 below for more details. As a consequence we have the following precise situation:

i) if $\exists \mu \in \sigma\left(A_{-1}\right)$ such that $|\mu|>1$ then a regular feedback cannot realize asymptotic stability;

ii) if for all $\mu \in \sigma\left(A_{-1}\right),|\mu| \leq 1$ and there exists $\mu$ such that $|\mu|=1$, then a regular feedback cannot realize exponential stabilizability;

iii) moreover, if for some $\mu \in \sigma\left(A_{-1}\right),|\mu|=1$ there exists a Jordan chain for this value, asymptotic stability cannot also be realized by a regular feedback.

Hence the initial case is when for all $\mu \in \sigma\left(A_{-1}\right)$, we have $|\mu| \leq 1$, and if $|\mu|=1$, then all Jordan blocks corresponding to this eigenvalue are of dimension 1, i.e. $\mu$ is a simple eigenvalue. As it was shown in [18] in this case asymptotic stability may appear or not. Roughly speaking the problem of strong stabilizability in our sense is to stabilize a neutral type system in this critical case by a regular feedback. We consider the case of a system (2) such that for all $\mu \in \sigma\left(A_{-1}\right),|\mu|=1$, the algebraic multiplicity of $\mu$ is 1 , and then we have for each such $\mu$ a Jordan block of dimension 1 . In this case, as the regular feedback does not change the matrix $A_{-1}$, then from the results proved in [18] it follows that the closed loop system will be asymptotically stable if and only if all the eigenvalues of $\mathcal{A}+\mathcal{B F}$ are in the left half plane: $\operatorname{Re} \sigma(\mathcal{A}+\mathcal{B F})<0$. Hence, the problem of regular stabilizability, in this case, consists in assigning the spectrum of the system in the left half plane. This is not typical for infinite dimensional systems, because the quality (location) of the spectrum is not enough to describe the behavior of solutions.

The main contribution of this paper is that, under some controllability conditions on the unstable poles of the system, we can assign arbitrarily the eigenvalues of the closed loop system into circles centered at the unstable eigenvalues of the operator $\mathcal{A}$ with radii $r_{k}$ such that $\sum r_{k}^{2}<\infty$. This is a generalization of the classical pole assignment problem in finite dimensional space (see also $[20,21]$ for an abstract point of view for infinite dimensional systems).

Precisely we have the following

Theorem 1 Consider the system (2) under the following assumptions:

1) All the eigenvalues of the matrix $A_{-1}$ satisfy $|\mu| \leq 1$.

2) All the eigenvalues $\mu_{j} \in \sigma_{1} \stackrel{\text { def }}{=} \sigma\left(A_{-1}\right) \cap\{z:|z|=1\}$ are simple (we denote their index $j \in I$ ). 
Then the system (2) is regularly asymptoticly stabilizable if

3) $\operatorname{rank}\left(\Delta_{\mathcal{A}}(\lambda) \quad B\right)=n$ for all $\operatorname{Re} \lambda \geq 0$, where

$$
\Delta_{\mathcal{A}}(\lambda)=-\lambda I+\lambda \mathrm{e}^{-\lambda} A_{-1}+\lambda \int_{-1}^{0} \mathrm{e}^{\lambda s} A_{2}(s) \mathrm{d} s+\int_{-1}^{0} \mathrm{e}^{\lambda s} A_{3}(s) \mathrm{d} s,
$$

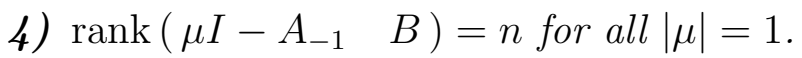

The preliminary version of these results for the case of one-dimensional control was presented in [19].

\subsection{Formulation of the problem}

Our purpose is to investigate the stabilization problem of the control system (2). This means to find a feedback control law which makes the closed loop system asymptotically stable. Depending on the sense of the needed stability and the property of feedback, one can find different senses of stabilizability. Our goal is to investigate the stabilizability in the following sense.

Definition 2 The system (2) (and (6)) is said to be regularly asymptoticly stabilizable (or regularly strongly stabilizable) if there exists a linear relatively $\mathcal{A}$-bounded feedback $u=\mathcal{F} x$ such that the operator $\mathcal{A}+\mathcal{B F}$ is such that $\mathcal{D}(\mathcal{A})=$ $\mathcal{D}(\mathcal{A}+\mathcal{B} \mathcal{F})$ and is the infinitesimal generator of a strongly asymptotically stable $C_{0}$-semigroup $\mathrm{e}^{(\mathcal{A}+\mathcal{B F}) t}$ in $M_{2}$.

Let us recall that the system $\left(\mathrm{e}^{\mathcal{A} t}, M_{2}\right)$, (without control) is said strongly asymptotically stable if

$$
\forall x=(y, z(\cdot)) \in M_{2}, \quad \lim _{t \rightarrow+\infty}\left\|\mathrm{e}^{\mathcal{A} t} x\right\|_{M_{2}}=0 .
$$

This problem has been investigated in details in [18] and we use here the results obtained there. In the sequel we use the fact that the last condition is equivalent to the condition $\left\|\mathrm{e}^{(\mathcal{A}+\mathcal{B} \mathcal{F}) t} x\right\|_{\mathcal{D}(\mathcal{A})} \rightarrow 0$, as $t \rightarrow \infty$ for all $x \in \mathcal{D}(\mathcal{A})$, where $\|\cdot\|_{\mathcal{D}(\mathcal{A})}$ is the $\mathcal{A}$-graph norm. Namely, we have the following general well known Lemma. The proof is given for the completeness of the presentation.

Lemma 3 Let $\mathrm{e}^{\mathcal{A} t}$ be a $C_{0}$-semigroup in a Hilbert space $X$. The following two conditions are equivalent

i) $\left\|\mathrm{e}^{\mathcal{A} t} x_{0}\right\| \rightarrow 0$ as $t \rightarrow \infty$ for all $x_{0} \in X$,

ii) $\left\|\mathrm{e}^{\mathcal{A} t} x_{0}\right\|_{\mathcal{D}(\mathcal{A})} \rightarrow 0$ as $t \rightarrow \infty$ for all $x_{0} \in \mathcal{D}(\mathcal{A})$. 
Proof. Let us remark that for $x_{0} \in \mathcal{D}(\mathcal{A})$ we have

$$
\left\|\mathrm{e}^{\mathcal{A} t} x_{0}\right\|_{\mathcal{D}(\mathcal{A})}=\left\|\mathrm{e}^{\mathcal{A} t} x_{0}\right\|+\left\|\mathcal{A} \mathrm{e}^{\mathcal{A} t} x_{0}\right\|=\left\|\mathrm{e}^{\mathcal{A} t} x_{0}\right\|+\left\|\mathrm{e}^{\mathcal{A} t} \mathcal{A} x_{0}\right\| .
$$

Then if we suppose that $\left\|\mathrm{e}^{\mathcal{A} t} x\right\| \rightarrow 0$ as $t \rightarrow \infty$ for all $x \in X$, this gives

$$
\left\|\mathrm{e}^{\mathcal{A} t} x_{0}\right\|_{\mathcal{D}(\mathcal{A})}=\left\|\mathrm{e}^{\mathcal{A} t} x_{0}\right\|+\left\|\mathrm{e}^{\mathcal{A} t} \mathcal{A} x_{0}\right\| \rightarrow 0
$$

Then i) implies ii).

Suppose now that $\left\|\mathrm{e}^{\mathcal{A} t} x_{0}\right\|_{\mathcal{D}(\mathcal{A})} \rightarrow 0$ as $t \rightarrow \infty$ for all $x_{0} \in \mathcal{D}(\mathcal{A})$. Then $\mathrm{e}^{\mathcal{A} t} x_{0}$ is uniformly bounded in $\mathcal{D}(\mathcal{A})$ :

$$
\left\|\mathrm{e}^{\mathcal{A} t} x_{0}\right\|_{\mathcal{D}(\mathcal{A})} \leq M\left\|x_{0}\right\|_{\mathcal{D}(\mathcal{A})} .
$$

One can without lost of generality suppose that $\|\mathcal{A} x\| \geq \alpha\|x\|$, i.e. $\mathcal{A}$ has a bounded inverse, if not we consider the equivalent norm

$$
\left\|x_{0}\right\|+\left\|\left(\lambda_{0} I-\mathcal{A}\right)^{-1} x_{0}\right\|, \quad \lambda_{0} \in \rho(\mathcal{A}) .
$$

Then

$$
\left\|\mathrm{e}^{\mathcal{A} t} x_{0}\right\|_{\mathcal{D}(\mathcal{A})} \leq M\left(\left\|x_{0}\right\|+\left\|\mathcal{A} x_{0}\right\|\right) \leq M\left(\frac{1}{\alpha}+1\right)\left\|\mathcal{A} x_{0}\right\|
$$

and

$$
\left\|\mathrm{e}^{\mathcal{A} t} \mathcal{A} x_{0}\right\|=\left\|\mathcal{A} \mathrm{e}^{\mathcal{A} t} x_{0}\right\| \leq\left\|\mathrm{e}^{\mathcal{A} t} x_{0}\right\|_{\mathcal{D}(\mathcal{A})} \leq M\left(\frac{1}{\alpha}+1\right)\left\|\mathcal{A} x_{0}\right\| .
$$

Putting $\mathcal{A} x_{0}=x$ this means that $\mathrm{e}^{\mathcal{A} t} x$ is bounded in $X$.

Now assume that $\left\|\mathrm{e}^{\mathcal{A} t} x_{0}\right\|_{\mathcal{D}(\mathcal{A})} \rightarrow 0$, as $t \rightarrow \infty$ for all $x_{0} \in \mathcal{D}(\mathcal{A})$. This means that $\left\|\mathrm{e}^{\mathcal{A} t} x_{0}\right\| \rightarrow 0$, for all $x_{0} \in \mathcal{D}(\mathcal{A})$. As $\left\|\mathrm{e}^{\mathcal{A} t} x_{0}\right\|$ is uniformly bounded in $\mathcal{D}(\mathcal{A})$, then $\left\|\mathrm{e}^{\mathcal{A} t} x_{0}\right\| \rightarrow 0$, as $t \rightarrow \infty \forall x_{0} \in X$.

To satisfy the condition $\mathcal{D}(\mathcal{A}+\mathcal{B F})=\mathcal{D}(\mathcal{A})$ it is enough for the term $\mathcal{B F}$ to be bounded with respect to $\mathcal{A}$ (see for example [8]). This means that the control may include the term of the form

$$
u=\int_{-1}^{0} F_{2}(\theta) \dot{z}_{t}(\theta) \mathrm{d} \theta+\int_{-1}^{0} F_{3}(\theta) z_{t}(\theta) \mathrm{d} \theta+F_{1} y
$$

for all $x=(y, z(\cdot)) \in \mathcal{D}(\mathcal{A})$. Here $F_{2}(\cdot), F_{3}(\cdot) \in L_{2}\left(-1,0 ; \mathbb{C}^{n \times p}\right), F_{1} \in \mathbb{C}^{n \times p}$.

Let us substitute the above control in (2):

$$
\begin{aligned}
\dot{z}(t)= & A_{-1} \dot{z}(t-1)+\int_{-1}^{0}\left[A_{2}(\theta)+B F_{2}(\theta)\right] \dot{z}_{t}(\theta) \mathrm{d} \theta \\
& +\int_{-1}^{0}\left[A_{3}(\theta)+B F_{3}(\theta)\right] z_{t}(\theta) \mathrm{d} \theta+B F_{1}\left[z_{t}(0)-A_{-1} z_{t}(-1)\right] .
\end{aligned}
$$


To rewrite the last term we use the formula

$$
f(0) z(0)-f(-1) z_{t}(-1)=\int_{-1}^{0} f(\theta) \dot{z}_{t}(\theta) \mathrm{d} \theta+\int_{-1}^{0} \dot{f}(\theta) z_{t}(\theta) \mathrm{d} \theta
$$

with

$$
f(\theta) \stackrel{\text { def }}{=} B F_{1}\left[I(\theta+1)-A_{-1} \theta\right]
$$

to get

$$
\begin{aligned}
& B F_{1}\left(z_{t}(0)-A_{-1} z_{t}(-1)\right)= \\
& \quad B F_{1}\left(\int_{-1}^{0}\left[I(\theta+1)-A_{-1} \theta\right] \dot{z}_{t}(\theta) \mathrm{d} \theta+\int_{-1}^{0}\left[I-A_{-1}\right] z_{t}(\theta)\right) .
\end{aligned}
$$

Substituting this into (11) we arrive to the following closed-loop system

$$
\begin{gathered}
\dot{z}(t)=A_{-1} \dot{z}(t-1)+\int_{-1}^{0}\left\{A_{2}(\theta)+B F_{2}(\theta)+B F_{1}\left[I(\theta+1)-A_{-1} \theta\right]\right\} \dot{z}_{t}(\theta) \mathrm{d} \theta \\
+\int_{-1}^{0}\left\{A_{3}(\theta)+B F_{3}(\theta)+B F_{1}\left[I-A_{-1}\right]\right\} z_{t}(\theta) \mathrm{d} \theta .
\end{gathered}
$$

Finally we obtain

$$
\dot{z}(t)=A_{-1} \dot{z}(t-1)+\int_{-1}^{0} \widetilde{A}_{2}(\theta) \dot{z}(t+\theta) \mathrm{d} \theta+\int_{-1}^{0} \widetilde{A}_{3}(\theta) z(t+\theta) \mathrm{d} \theta,
$$

where

$$
\begin{aligned}
& \widetilde{A}_{2}(\theta) \stackrel{\text { def }}{=} A_{2}(\theta)+B F_{2}(\theta)+B F_{1}\left[I(\theta+1)-A_{-1} \theta\right], \\
& \widetilde{A}_{3}(\theta) \stackrel{\text { def }}{=} A_{3}(\theta)+B F_{3}(\theta)+B F_{1}\left[I-A_{-1}\right] .
\end{aligned}
$$

Comparing (1) and (12) we can see that any control of the form (10) with $F_{2}(\cdot), F_{3}(\cdot) \in L_{2}\left(-1,0 ; \mathbb{C}^{n \times p}\right), F_{1} \in \mathbb{C}^{n \times p}$ transforms the control system (2) into the class of initial system (1), so we can use all the results of our investigations in [18]. It is important to notice that the term $A_{-1} \dot{z}(t-1)$ remains unchanged, since any perturbation $\left(A_{-1}+K\right) \dot{z}(t-1)$ immediately changes the domain of the operator (see (6)).

\subsection{The organization of the paper}

The Section 2 is devoted to the analysis of the spectrum of the operator model (6). This part precises some results given in [18]. We give also a description 
of the adjoint operator $\mathcal{A}^{*}$ and its eigenvectors, which play an important role in the formulation of the stabilizability result. Section 3 is concerned with the formulation and the proof of an abstract stabilizability result for systems in Hilbert spaces. This result is used for the detailed proof of the main result which is given in Section 5, when in Section 4 we give the formulation of the main result with a short proof. The technical Section 5 is divided into two parts. The first one is devoted to the proof of the assignment of an infinite part of the unstable spectrum, assumed to be controllable. The second part conclude the proof with moving the remaining finite unstable part. In the last Section we discuss the result and explain why the conditions of the main Theorem are essential and give remaining open problems.

\section{Spectral analysis}

In this section we present a result on the location of $\sigma(\mathcal{A})$ which improves Theorem 2 in [18] and we give the expressions of the adjoint operator $\mathcal{A}^{*}$ and their eigenvectors. These results an important role in our considerations.

\subsection{The location of the spectrum}

We will use notations of paragraph 2.2 in [18]. In the sequel we will consider the matrix $A_{-1}$ in a Jordan basis and change the norm in $\mathbb{C}^{n}$ such that the corresponding eigen- and rootvectors of $A_{-1}$ form an orthogonal basis. Let us denote by $\mu_{1}, \ldots, \mu_{\ell}, \mu_{i} \neq \mu_{j}$ if $i \neq j$, the eigenvalues of $A_{-1}$ and the dimensions of their rootspaces by $p_{1}, \ldots, p_{\ell}, \sum_{k=1}^{\ell} p_{k}=n$. Let $\nu_{m}$ be the number of Jordan blocks, corresponding to $\mu_{m} \in \sigma\left(A_{-1}\right)$. Denote by $p_{m, j}, j=1, . ., \nu_{m}$, the orders of these blocks, so $\sum_{j=1}^{\nu_{m}} p_{m, j}=p_{m}$. Consider the points

$$
\lambda_{m}^{(k)}=\ln \left|\mu_{m}\right|+\mathrm{i}\left(\arg \mu_{m}+2 \pi k\right), m=1, . ., \ell ; k \in \mathbb{Z}
$$

and the circles $L_{m}^{(k)} \stackrel{\text { def }}{=} L_{m}^{(k)}\left(r_{0}\right)$ of fixed radius $r_{0}=\frac{1}{3} \min \left\{\left|\lambda_{m}^{(k)}-\lambda_{i}^{(j)}\right|,(m, k) \neq\right.$ $(i, j)\}$ centered at $\lambda_{m}^{(k)}$. We will also need the circles $L_{m}^{(k)}\left(r^{(k)}\right)$ centered at $\lambda_{m}^{(k)}$ with radii $r^{(k)}$ to be specified later.

Let us introduce the values

$$
\begin{aligned}
F_{m}^{(k)} \stackrel{\text { def }}{=} \max \left\{\frac{1}{2 \pi|k|} \cdot \sup _{\widetilde{\lambda} \in L_{m}^{(0)}\left(r_{0}\right)}\left\|\int_{-1}^{0} \mathrm{e}^{\mathrm{i} \cdot 2 \pi k}\left(A_{2}(s) \mathrm{e}^{\widetilde{\lambda} s}\right) \mathrm{d} s\right\| ;\right. \\
\left.\frac{1}{2 \pi|k|} \cdot \underset{\widetilde{\lambda} \in L_{m}^{(0)}\left(r_{0}\right)}{\sup _{-1}}\left\|\int_{-1}^{0} \mathrm{e}^{\mathrm{i} \cdot 2 \pi k}\left(A_{3}(s) \mathrm{e}^{\widetilde{\lambda} s}\right) \mathrm{d} s\right\|\right\} .
\end{aligned}
$$


It is important to notice that each supremum is taken over the circles $L_{m}^{(0)}\left(r_{0}\right)$ for all values of the index $k$.

Let us discuss the properties of $F_{m}^{(k)}$. The property $A_{i} \in L_{2}(-1,0),(i=2,3)$ implies that for any fixed $\widetilde{\lambda} \in L_{m}^{(0)}\left(r_{0}\right)$ the functions $A_{i}(s) \mathrm{e}^{\widetilde{\lambda} s}, i=2,3$, belong to $L_{2}(-1,0)$ and do not depend on $k$. The functions $\left\{\mathrm{e}^{\mathrm{i} \cdot 2 \pi k}\right\}_{k \in \mathbb{Z}}$ form the trigonometric basis of $L_{2}(-1,0)$. So the integrals in (13) are the Fourier coefficients of $A_{i}(s) \mathrm{e}^{\widetilde{\lambda} s}$. It implies the estimate which plays an essential role in the sequel

$$
\sum_{k \in \mathbb{Z}}\left(F_{m}^{(k)}\right)^{2}<\infty \quad \text { for all } m
$$

We also denote

$$
\Delta_{\mathcal{A}}(\lambda)=-\lambda I+\lambda \mathrm{e}^{-\lambda} A_{-1}+\lambda \int_{-1}^{0} \mathrm{e}^{\lambda s} A_{2}(s) \mathrm{d} s+\int_{-1}^{0} \mathrm{e}^{\lambda s} A_{3}(s) \mathrm{d} s .
$$

The following theorem improves Theorem 2 in [18].

Theorem 4 The spectrum of $\mathcal{A}$ consists of the eigenvalues only which are the roots of the equation $\operatorname{det} \Delta_{\mathcal{A}}(\lambda)=0$, where $\Delta_{\mathcal{A}}(\lambda)$ is given by (15). The corresponding eigenvectors of $\mathcal{A}$ are $\varphi=\left(\begin{array}{c}C-\mathrm{e}^{-\lambda} A_{-1} C \\ \mathrm{e}^{\lambda \cdot C} C\end{array}\right)$, with $C \in \operatorname{Ker} \Delta_{\mathcal{A}}(\lambda)$. There exists $N_{1}$ such that for any $k$, satisfying $|k| \geq N_{1}$, the total multiplicity of the roots of the equation $\operatorname{det} \Delta_{\mathcal{A}}(\lambda)=0$, contained in the circles $L_{m}^{(k)}\left(r^{(k)}\right)$, equals $p_{m}$, where radii $r^{(k)}$ satisfy

$$
\sum_{k \in \mathbb{Z}}\left(r^{(k)}\right)^{2 p_{m}^{\max }}<\infty
$$

Here $p_{m}^{\max } \stackrel{\text { def }}{=} \max \left\{p_{m, j}, j=1, . ., \nu_{m}\right\}$ the maximal order of the Jordan blocks, corresponding to $\mu_{m} \in \sigma\left(A_{-1}\right)$.

Moreover, we can take different radii $r_{m}^{(k)}$ for different circles $L_{m}^{(k)}\left(r_{m}^{(k)}\right)$, such that $\left(r_{m}^{(k)}\right)^{p_{m}^{\max }}$ are proportional to $F_{m}^{(k)}$ (see (13)) i.e. there exists $d_{1}>0$, such that for any $|k| \geq N_{1}$, we have $\left(r_{m}^{(k)}\right)^{p_{m}^{\max }}=d_{1} \cdot F_{m}^{(k)}$.

Corollary 5 If $\mu_{m}$ is a simple root, i.e. $p_{m}^{\max }=1$, we have $\sum_{k \in \mathbb{Z}}\left(r^{(k)}\right)^{2}<\infty$.

Remark 6 Sometimes, for simplicity, we will take in (16) $p_{m}$ instead of $p_{m}^{\max }$ since $p_{m}^{\max } \leq p_{m} \stackrel{\text { def }}{=} \sum_{j=1}^{\nu_{m}} p_{m, j}$.

Proof of Theorem 4. We follow line of argument of the proof of Theorem 2 
in [18] and describe the location of the spectrum of $\mathcal{A}$ using Rouché theorem. Let us introduce the functions

$$
\begin{aligned}
f_{1}(\lambda) \stackrel{\text { def }}{=} & \operatorname{det}\left(A_{-1}-\mathrm{e}^{\lambda} I\right) \\
f_{2}(\lambda) \stackrel{\text { def }}{=} & \operatorname{det}\left(A_{-1}-\mathrm{e}^{\lambda} I\right) \\
& \quad-\operatorname{det}\left(\left(A_{-1}-\mathrm{e}^{\lambda} I\right)+\mathrm{e}^{\lambda} \int_{-1}^{0} \mathrm{e}^{\lambda s} A_{2}(s) \mathrm{d} s+\mathrm{e}^{\lambda} \lambda^{-1} \int_{-1}^{0} \mathrm{e}^{\lambda s} A_{3}(s) \mathrm{d} s\right) .
\end{aligned}
$$

We will show that $\left|f_{1}(\lambda)\right|>\left|f_{2}(\lambda)\right|$ for any $\lambda \in L_{m}^{(k)}, m=1, \ldots, \ell$, when $k$ is sufficiently large. Thus, $f_{1}-f_{2}$ have the same number of roots inside $L_{m}^{(k)}$ as function $f_{1}$ has. On the other hand the roots of $f_{1}(\lambda)-f_{2}(\lambda)$ are the same as the roots of $\operatorname{det} \Delta_{\mathcal{A}}(\lambda)$ for $\lambda \in L_{m}^{(k)}$ and for sufficiently large $k$. Let us rewrite $f_{2}$ as follows:

$$
f_{2}(\lambda)=\operatorname{det}\left(A_{-1}-\mathrm{e}^{\lambda} I\right)\left[1-\operatorname{det}\left(I+\left(A_{-1}-\mathrm{e}^{\lambda} I\right)^{-1} L(\lambda)\right)\right],
$$

where

$$
L(\lambda)=\mathrm{e}^{\lambda} \int_{-1}^{0} \mathrm{e}^{\lambda s} A_{2}(s) \mathrm{d} s+\mathrm{e}^{\lambda} \lambda^{-1} \int_{-1}^{0} \mathrm{e}^{\lambda s} A_{3}(s) \mathrm{d} s .
$$

To show that $\left|f_{1}(\lambda)\right|>\left|f_{2}(\lambda)\right|$ it is sufficient to get

$$
\left|1-\operatorname{det}\left(I+\left(A_{-1}-\mathrm{e}^{\lambda} I\right)^{-1} L(\lambda)\right)\right|<1 .
$$

To this end we show the inequality

$$
\left.\|\left(A_{-1}-\mathrm{e}^{\lambda} I\right)^{-1} L(\lambda)\right) \| \leq \nu
$$

for sufficiently small $\nu$ (and all large $k$ ) which gives (18) (for details see [18]). Since $\left.\left.\|\left(A_{-1}-\mathrm{e}^{\lambda} I\right)^{-1} L(\lambda)\right)\|\leq\|\left(A_{-1}-\mathrm{e}^{\lambda} I\right)^{-1}\|\| L(\lambda)\right) \|$ we need two estimates:

$$
\left\|\left(A_{-1}-\mathrm{e}^{\lambda} I\right)^{-1}\right\| \leq \frac{C_{1}}{\left(r^{(k)}\right)^{p_{m}^{\max }}}
$$

for some $C_{1}>0$ and

$$
\left.\forall \lambda \in L_{m}^{(k)}\left(r^{(k)}\right), \quad \| L(\lambda)\right) \| \leq \alpha_{m, k}, \quad \sum_{k \in \mathbb{Z}} \alpha_{m, k}^{2}<\infty .
$$

We have $\lambda=\lambda_{m}^{(k)}+r^{(k)} \mathrm{e}^{i \phi}, \phi \in[0,2 \pi]$, hence

$$
\left|\mu_{m}-\mathrm{e}^{\lambda}\right|=\left|\mu_{m}-\mu_{m} \mathrm{e}^{r^{(k)} \mathrm{e}^{i \phi}}\right|=\left|\mu_{m}\right| \cdot\left|1-\mathrm{e}^{r^{(k)} \mathrm{e}^{i \phi}}\right| \geq C_{0} \cdot r^{(k)}>0
$$


for all $m, k, \lambda \in L_{m}^{(k)}$ and $C_{0}$ independent of $m$ and $\lambda \in L_{m}^{(k)}$. We use here the assumption that $\operatorname{det} A_{-1} \neq 0$ which implies $\min \left|\mu_{m}\right|>0$. Using the fact that $A_{-1}$ has a Jordan form and a well known fact that for a Jordan block $J$ of dimension $d_{J}$, corresponding to the eigenvalue $\mu$, one has for $R_{J}\left(\mathrm{e}^{\lambda}\right)=$ $\left(J-\mathrm{e}^{\lambda} I\right)^{-1}$ the expression:

$$
R_{\mathrm{e}^{\lambda}}(J)=\left(\begin{array}{cccc}
\left(\mu-\mathrm{e}^{\lambda}\right)^{-1} & -\left(\mu-\mathrm{e}^{\lambda}\right)^{-2} & \cdots & (-1)^{d_{J}-1}\left(\mu-\mathrm{e}^{\lambda}\right)^{-d_{J}} \\
0 & \left(\mu-\mathrm{e}^{\lambda}\right)^{-1} & \cdots & (-1)^{d_{J}-2}\left(\mu-\mathrm{e}^{\lambda}\right)^{-d_{J}+1} \\
\vdots & \vdots & \ddots & \vdots \\
0 & 0 & \cdots & \left(\mu-\mathrm{e}^{\lambda}\right)^{-1}
\end{array}\right)
$$

and we deduce $(20)$ taking $d_{J}=p_{m}^{\max }$.

To obtain (21), we need to estimate $\| L(\lambda)) \|$. We write $\lambda \in L_{m}^{(k)}\left(r^{(k)}\right)$ as $\lambda=\widetilde{\lambda}+\mathrm{i} \cdot 2 \pi k$, with $\widetilde{\lambda} \in L_{m}^{(0)}\left(r_{0}\right)$. Now it is enough to consider

$$
\int_{-1}^{0} \mathrm{e}^{\lambda s} A_{i}(s) \mathrm{d} s=\int_{-1}^{0} \mathrm{e}^{\mathrm{i} \cdot 2 \pi k}\left(A_{i}(s) \mathrm{e}^{\widetilde{\lambda} s}\right) \mathrm{d} s .
$$

It is easy to deduce from (13), (17), and (23) that for any $\lambda \in L_{m}^{(k)}\left(r^{(k)}\right)$ with $r^{(k)} \leq r_{0}$ one has

$$
\|L(\lambda)\| \leq \alpha_{m, k} \stackrel{\text { def }}{=} d_{0} F_{m}^{(k)}, \quad d_{0}>0
$$

So estimate (21) follows from (14).

Let us choose small enough value of $\nu$ (see (19)). Estimate (19) is satisfied provided (see (20), (21) and (24))

$$
\frac{C_{1} \alpha_{m, k}}{\left(r^{(k)}\right)^{p_{m}^{\max }}}=\frac{C_{1} d_{0} F_{m}^{(k)}}{\left(r^{(k)}\right)^{p_{m}^{\max }}} \leq \nu
$$

The last inequality is satisfied if we choose, for example,

$$
\left(r^{(k)}\right)^{p_{m}^{\max }}=F_{m}^{(k)} C_{1} d_{0} \nu^{-1}=F_{m}^{(k)} d_{1}, \quad d_{1} \stackrel{\text { def }}{=} C_{1} d_{0} \nu^{-1} .
$$

Finally, under the above assumptions, estimate (19) allows us to apply Rouché theorem and completes the proof of Theorem 4 . 


\subsection{The adjoint operator $\mathcal{A}^{*}$}

We give now the expression of the adjoint operator for $\mathcal{A}$. Of special interest is the characterization of the eigenvectors of $\mathcal{A}^{*}$ to be used in the next sections.

Theorem 7 The adjoint operator $\mathcal{A}^{*}$ is given by

$$
\mathcal{A}^{*}\left(\begin{array}{c}
y \\
\psi(\cdot)
\end{array}\right)=\left(\begin{array}{c}
A_{2}^{*}(0) y+\psi(0) \\
-\frac{\mathrm{d}}{\mathrm{d} \theta}\left[\psi(\theta)+A_{2}^{*}(\theta) y\right]+A_{3}^{*}(\theta) y
\end{array}\right),
$$

with the domain of the operator $\mathcal{A}^{*}$ being

$$
\begin{aligned}
\mathcal{D}\left(\mathcal{A}^{*}\right)=\left\{(y, \psi(\cdot)): \psi(\theta)+A_{2}^{*}(\theta) y \in H^{1}\left(-1,0 ; \mathbb{C}^{n}\right),\right. & \\
& \left.\left(A_{-1}^{*} A_{2}^{*}(0)-A_{2}^{*}(-1)\right) y=\psi(-1)-A_{-1}^{*} \psi(0)\right\} \subset M_{2} .
\end{aligned}
$$

The eigenvectors of $\mathcal{A}^{*}$ are given by

$$
\left(\begin{array}{c}
y \\
{\left[\lambda \mathrm{e}^{-\lambda \theta}-A_{2}^{*}(\theta)+\mathrm{e}^{-\lambda \theta} \int_{0}^{\theta} \mathrm{e}^{\lambda s} A_{3}^{*}(s) \mathrm{d} s+\lambda \mathrm{e}^{-\lambda \theta} \int_{0}^{\theta} \mathrm{e}^{\lambda s} A_{2}^{*}(s) \mathrm{d} s\right] y}
\end{array}\right),
$$

where $y \in \operatorname{Ker} \Delta_{\mathcal{A}}^{*}(\lambda)$ with

$$
\Delta_{\mathcal{A}}^{*}(\lambda) \stackrel{\text { def }}{=} \lambda \mathrm{e}^{-\lambda} A_{-1}^{*}-\lambda I+\lambda \int_{-1}^{0} \mathrm{e}^{\lambda s} A_{2}^{*}(s) \mathrm{d} s+\int_{-1}^{0} \mathrm{e}^{\lambda s} A_{3}^{*}(s) \mathrm{d} s .
$$

The eigenvalues are the roots of the equation $\operatorname{det} \Delta_{\mathcal{A}}^{*}(\lambda)=0$.

Proof. Using the definition of the operator $\mathcal{A}$ and after some calculations one can check that

$$
\begin{aligned}
\left\langle\mathcal{A}\left(\begin{array}{c}
v \\
z(\cdot)
\end{array}\right),\left(\begin{array}{c}
y \\
\psi(\cdot)
\end{array}\right)\right\rangle_{M_{2}}= & \left\langle\int_{-1}^{0} A_{2}(\theta) \dot{z}(\theta) \mathrm{d} \theta+\int_{-1}^{0} A_{3}(\theta) z(\theta) \mathrm{d} \theta, y\right\rangle_{\mathbb{C}^{n}} \\
& +\int_{-1}^{0}\langle\dot{z}(\theta), \psi(\theta)\rangle_{\mathbb{C}^{n}} \mathrm{~d} \theta \\
= & \int_{-1}^{0}\left\langle\dot{z}(\theta), A_{2}^{*}(\theta) y+\psi(\theta)+\int_{\theta}^{0} A_{3}^{*}(s) y \mathrm{~d} s\right\rangle_{\mathbb{C}^{n}} \mathrm{~d} \theta \\
& +\int_{-1}^{0}\left\langle z(-1), A_{3}^{*}(\theta) y\right\rangle_{\mathbb{C}^{n}} \mathrm{~d} \theta
\end{aligned}
$$

Our goal is to find $\left(\begin{array}{c}h \\ w(\cdot)\end{array}\right)$ such that

$$
\left\langle\mathcal{A}\left(\begin{array}{c}
v \\
z(\cdot)
\end{array}\right),\left(\begin{array}{c}
y \\
\psi(\cdot)
\end{array}\right)\right\rangle_{M_{2}}=\left\langle\left(\begin{array}{c}
v \\
z(\cdot)
\end{array}\right),\left(\begin{array}{c}
h \\
w(\cdot)
\end{array}\right)\right\rangle_{M_{2}}
$$


then $\mathcal{A}^{*}\left(\begin{array}{c}y \\ \psi(\cdot)\end{array}\right)=\left(\begin{array}{c}h \\ w(\cdot)\end{array}\right)$. As $\left(\begin{array}{c}v \\ z(\cdot)\end{array}\right) \in \mathcal{D}(\mathcal{A})$ we get

$$
\begin{aligned}
\left\langle\left(\begin{array}{c}
v \\
z(\cdot)
\end{array}\right),\left(\begin{array}{c}
h \\
w(\cdot)
\end{array}\right)\right\rangle_{M_{2}} & =\langle v, h\rangle_{\mathbb{C}^{n}}+\int_{-1}^{0}\langle z(\theta), w(\theta)\rangle_{\mathbb{C}^{n}} \mathrm{~d} \theta \\
& =\langle z(0), h\rangle_{\mathbb{C}^{n}}-\left\langle z(-1), A_{-1}^{*} h\right\rangle_{\mathbb{C}^{n}}+\int_{-1}^{0}\langle z(\theta), w(\theta)\rangle_{\mathbb{C}^{n}} \mathrm{~d} \theta
\end{aligned}
$$

and this gives

$$
\begin{aligned}
& \left\langle\left(\begin{array}{c}
v \\
z(\cdot)
\end{array}\right),\left(\begin{array}{c}
h \\
w(\cdot)
\end{array}\right)\right\rangle_{M_{2}}= \\
& \int_{-1}^{0}\left\langle\dot{z}(\theta), \int_{\theta}^{0} w(s) \mathrm{d} s+h\right\rangle_{\mathbb{C}^{n}} \mathrm{~d} \theta+\left\langle z(-1), h-A_{-1}^{*} h+\int_{-1}^{0} w(\theta) \mathrm{d} \theta\right\rangle_{\mathbb{C}^{n}} .
\end{aligned}
$$

Substituting this relation and (29) into (30) we select the corresponding terms with $\dot{z}(\theta)$ and $z(-1)$ to get

$$
\int_{\theta}^{0} w(s) \mathrm{d} s+h=A_{2}^{*}(\theta) y+\psi(\theta)+\int_{\theta}^{0} A_{3}^{*}(s) y \mathrm{~d} s
$$

and

$$
h-A_{-1}^{*} h+\int_{-1}^{0} w(\theta) \mathrm{d} \theta=\int_{-1}^{0} A_{3}^{*}(\theta) y \mathrm{~d} \theta .
$$

The assumption $A_{3}^{*}(\cdot), w(\cdot) \in L_{2}\left(-1,0 ; \mathbb{C}^{n}\right)$ and (31) imply

$$
A_{2}^{*}(\cdot) y+\psi(\cdot) \in H^{1}\left([-1,0] ; \mathbb{C}^{n}\right) .
$$

The last property allows to take $\theta=0$ and then $\theta=-1$ in (31) to obtain

$$
h=A_{2}^{*}(0) y+\psi(0), \quad A_{-1}^{*} h=A_{2}^{*}(-1) y+\psi(-1) .
$$

The property (33) allows to differentiate the equality (31) to get

$$
w(\theta)=-\frac{\mathrm{d}}{\mathrm{d} \theta} \psi(\theta)-\frac{\mathrm{d}}{\mathrm{d} \theta} A_{2}^{*}(\theta) y+A_{3}^{*}(\theta) y .
$$

As we mentioned, $\mathcal{A}^{*}\left(\begin{array}{c}y \\ \psi(\cdot)\end{array}\right)=\left(\begin{array}{c}h \\ w(\cdot)\end{array}\right)$. Hence (34) and (35) give the formula for the operator $\mathcal{A}^{*}$ and its domain presented in (25) and (26). 
To get the form of an eigenvector we write its definition $\mathcal{A}^{*}\left(\begin{array}{c}y \\ \psi(\cdot)\end{array}\right)=\lambda\left(\begin{array}{c}y \\ \psi(\cdot)\end{array}\right)$, with $\left(\begin{array}{c}y \\ \psi(\cdot)\end{array}\right) \in \mathcal{D}\left(\mathcal{A}^{*}\right)$ which implies

$$
A_{2}^{*}(0) y+\psi(0)=\lambda y
$$

and

$$
-\frac{\mathrm{d}}{\mathrm{d} \theta} \psi-\frac{\mathrm{d}}{\mathrm{d} \theta} A_{2}^{*}(\theta) y+A_{3}^{*}(\theta) y=\lambda \psi(\theta) .
$$

From (37) we obtain

$$
\psi(\theta)=\mathrm{e}^{-\lambda \theta} \psi(0)+\int_{0}^{\theta} \mathrm{e}^{-\lambda(\theta-s)}\left(A_{3}^{*}(s)-\frac{\mathrm{d}}{\mathrm{d} \theta} A_{2}^{*}(s)\right) \mathrm{d} s \cdot y
$$

and using the expression of $\psi(0)$ from (36) we infer

$$
\psi(\theta)=\left[\mathrm{e}^{-\lambda \theta} \lambda I-\mathrm{e}^{-\lambda \theta} A_{2}^{*}(0)+\int_{0}^{\theta} \mathrm{e}^{-\lambda(\theta-s)}\left(A_{3}^{*}(s)-\dot{A}_{2}^{*}(s)\right) \mathrm{d} s\right] y
$$

Calculations give the formula for $\psi(\theta)$ presented in the second line of (27) and the form of the eigenvector. From (27) we deduce

$$
\psi(-1)=\left[\lambda \mathrm{e}^{\lambda} I-A_{2}^{*}(-1)-\mathrm{e}^{\lambda} \int_{-1}^{0} \mathrm{e}^{\lambda s} A_{3}^{*}(s) \mathrm{d} s-\lambda \mathrm{e}^{\lambda} \int_{-1}^{0} \mathrm{e}^{\lambda s} A_{2}^{*}(s) \mathrm{d} s\right] y
$$

and substitute it in (26) to get the formula for $\Delta_{\mathcal{A}}^{*}(\lambda)$ (see (28)). This completes the proof of Theorem 7 .

\section{Abstract problem of infinite pole assignment}

In this section we present an abstract approach to the stabilization problem for a general operator model and this approach will be used in the next sections to stabilize the neutral type system.

The approach presented here is a generalization of the idea proposed in [20] for the problem of infinite pole assignment for the case of the wave (partial differential) equation. In this case, the operator under consideration is skewadjoint with a simple spectrum while in the present case it satisfies neither first nor second assumption. Nevertheless, the main idea of [20], after necessary 
improvements, allows us to treat more general cases including neutral type operator model.

Here we use the notation $\mathcal{A}$ for an operator satisfying the assumptions given below. As it will be shown in the next section, the operator defined in (6) satisfies these assumptions, so the reader mainly interested in the neutral type system may simply look at $\mathcal{A}$ as at the operator (6).

As before, we denote the points

$$
\lambda_{m}^{(k)}=\ln \left|\mu_{m}\right|+\mathrm{i}\left(\arg \mu_{m}+2 \pi k\right), m=1, . ., \ell ; k \in \mathbb{Z}
$$

and the circles $L_{m}^{(k)}\left(r^{(k)}\right)$ centered at $\lambda_{m}^{(k)}$ with radii $r^{(k)}$, satisfying

$$
\sum_{k \in \mathbb{Z}}\left(r^{(k)}\right)^{2}<\infty
$$

Let $H$ be a complex Hilbert space. We consider an infinitesimal generator $\mathcal{A}$ of a $C_{0}$-semigroup in $H$ with domain $\mathcal{D}(\mathcal{A}) \subset H$. We have the following assumptions:

H1) The spectrum of $\mathcal{A}$ consists of the eigenvalues only which are located in the circles $L_{m}^{(k)}\left(r^{(k)}\right)$, where radii $r^{(k)}$ satisfy (38). Moreover, there exists $N_{1}$ such that for any $k$, satisfying $|k| \geq N_{1}$, the total multiplicity of the eigenvalues, contained in the circles $L_{m}^{(k)}\left(r^{(k)}\right)$, equals $p_{m} \in \mathbb{N}$, i.e. the multiplicity is finite and does not depend on $k$.

We need the spectral projectors

$$
P_{m}^{(k)}=\frac{1}{2 \pi \mathrm{i}} \int_{L_{m}^{(k)}} R(\mathcal{A}, \lambda) \mathrm{d} \lambda
$$

to define the subspaces $V_{m}^{(k)}=P_{m}^{(k)} H$.

H2) There exists a sequence of invariant for operator $\mathcal{A}$ finite-dimensional subspaces which constitute a Riesz basis in $H$. More precisely, there exists $N_{0}$ large enough, such that for any $N \geq N_{0}$, these subspaces are $\left\{V_{m}^{(k)}\right\}_{\substack{|k| \geq N \\ m=1, \ldots, \ell}}$ and $W_{N}$, where the last one is the $2(N+1) n$-dimensional subspace spanned by all eigen- and rootvectors, corresponding to all eigenvalues of $\mathcal{A}$, which are outside of all circles $L_{m}^{(k)},|k| \geq N, m=1, . ., \ell$.

The scalar product and the norm in which all the finite-dimensional subspaces $V_{m}^{(k)}$ and $W_{N}$ are orthogonal and form a Riesz basis of subspaces are denoted by $\langle\cdot, \cdot\rangle_{0}$ and $\|\cdot\|_{0}$. 
H3) The system is of single input, i.e. the operator $\mathcal{B}: \mathbb{C} \rightarrow H$ is the operator of multiplication by $\mathbf{b} \in H$.

The main result of this section is the following

Theorem 8 (On infinite pole assignment). Assume the assumptions H1)H3) are satisfied. Consider an infinite set of circles $L_{m}^{(k)}\left(r^{(k)}\right)$ such that each $L_{m}^{(k)}\left(r^{(k)}\right)$ contains only one simple eigenvalue of $\mathcal{A}$ only, i.e. $p_{m}=1$. We denote the set of indexes of these circles by $m \in I$.

We assume that $\mathbf{b} \in H$ is not orthogonal to eigenvectors $\varphi_{m}^{k}$, of $\mathcal{A}^{*}$ for $m \in I$ i.e.

$$
\left\langle\mathbf{b}, \varphi_{m}^{k}\right\rangle_{0} \neq 0 \quad \text { for all } \quad|k| \geq N, m \in I
$$

and

$$
\lim _{k \rightarrow \infty} k \cdot\left|\left\langle\mathbf{b}, \varphi_{m}^{k}\right\rangle_{0}\right|=c_{m}, \in \mathbb{R} \quad \text { for all } \quad m \in I
$$

Then there exists $N_{2} \geq N$ such that for any family of complex numbers $\widetilde{\lambda}_{m}^{k} \in$ $L_{m}^{(k)}\left(r^{(k)}\right), m \in I,|k| \geq N_{2}$ there exists a linear control $\mathcal{F}: \mathcal{D}(\mathcal{A}) \rightarrow \mathbb{C}$, such that

1) the complex numbers $\widetilde{\lambda}_{m}^{k}$ are eigenvalues of the operator $\mathcal{A}+\mathcal{B F}$;

2) the operator $\mathcal{B F}: \mathcal{D}(\mathcal{A}) \rightarrow H$ is relatively $\mathcal{A}$-bounded.

Proof. By Assumption H2), the simplicity of the spectrum and the definition of scalar product $\langle\cdot, \cdot\rangle_{0}$, the eigenvectors $\left\{\varphi_{m}^{k}\right\} \quad(m \in I)$ of the operator $\mathcal{A}$ satisfy $\left\langle\varphi_{m}^{k}, \varphi_{i}^{j}\right\rangle_{0}=1$ iff $k=j, m=i$ and $\left\langle\varphi_{m}^{k}, \varphi_{i}^{j}\right\rangle_{0}=0$ otherwise. The corresponding eigenvalues are denoted by $\lambda_{m}^{k}$. Moreover, any vector $x \in H$ can be represented as follows

$$
x=\sum_{m \in I} \sum_{|k| \geq N} \varphi_{m}^{k} \cdot\left\langle x, \varphi_{m}^{k}\right\rangle_{0}+P x
$$

where $P$ is the orthogonal, with respect to in $\langle., .\rangle_{0}$, projector. We can write

$$
\|x\|_{0}^{2}=\sum_{m \in I} \sum_{|k| \geq N}\left\langle x, \varphi_{m}^{k}\right\rangle_{0}^{2}+\|P x\|_{0}^{2} .
$$

Let us introduce a new norm (non-equivalent to the norm $\|\cdot\|_{0}$ ) which is of prime importance for our approach:

$$
\|x\|_{1}^{2} \stackrel{\text { def }}{=} \sum_{m \in I} \sum_{|k| \geq N}\left(\xi_{m}^{k}\right)^{2} \cdot\left\langle x, \varphi_{m}^{k}\right\rangle_{0}^{2}+\|P x\|_{0}^{2},
$$


where the constants $\xi_{m}^{k}$ are such $\xi_{m}^{k}>0, \xi_{m}^{k} \rightarrow \infty$ as $k \rightarrow \infty$ and will be chosen more precisely later (in (44)).

Let us now consider the control $u=\mathcal{F} x \stackrel{\text { def }}{=}\langle x, f\rangle_{1}$, where the vector $f$ is to be chosen later. In other words, we look for a linear feedback control, bounded with respect to the norm $\|\cdot\|_{1}$.

Property (40) allows us to choose $\left\{\xi_{m}^{k}\right\}$ as

$$
\xi_{m}^{k} \stackrel{\text { def }}{=}\left|\left\langle\mathbf{b}, \varphi_{m}^{k}\right\rangle_{0}\right|^{-1} \quad \text { for all } \quad m \in I, \quad|k| \geq N
$$

where $\left\{\xi_{m}^{k}\right\}$ define the new norm $\|\cdot\|_{1}$ (see (43)), corresponding to the inner product $\langle., .\rangle_{1}$. We also choose $\widetilde{\lambda}_{m}^{k}$ such that

$$
\sum_{m \in I} \sum_{|k| \geq N}\left|\widetilde{\lambda}_{m}^{k}-\lambda_{m}^{(k)}\right|^{2}<+\infty
$$

where $\lambda_{m}^{(k)} \stackrel{\text { def }}{=} \ln \left|\mu_{m}\right|+i\left(\arg \mu_{m}+2 \pi k\right)$ (see also Paragraph 2.2 in [18]). The property (45) is equivalent to $\widetilde{\lambda}_{m}^{n} \in L_{m}^{(n)}\left(r^{(n)}\right), m \in I$ (see the statement of Theorem 8) together with (38).

The feedback low which allows to move the infinite family of eigenvalues is designed using a special choice of a vector in $H$. This vector is constructed according to some choice of new eigenvalues. This is based on the following

Lemma 9 Consider the vectors

$$
q_{m}^{k} \stackrel{\text { def }}{=} \frac{1}{\xi_{m}^{k}} \cdot \frac{\lambda_{m}^{k}-\widetilde{\lambda}_{m}^{k}}{\left\langle\mathbf{b}, \varphi_{m}^{k}\right\rangle_{0}} \cdot R_{\widetilde{\lambda}_{m}^{k}}(\mathcal{A}) b \in H .
$$

There exists an integer $N_{2}$ such that vectors $\left\{q_{m}^{k}\right\}_{m \in I,|k| \geq N_{2}}$ form a Riesz basis of the closure (in $\|\cdot\|_{1}$ ) of their linear span, say $Q H \stackrel{\text { def }}{=} \operatorname{Clspan}\left\{q_{m}^{k}: m \in\right.$ $\left.I,|k| \geq N_{2}\right\}$ (here we denoted by $Q$ the orthogonal, in $\|\cdot\|_{1}$, projector onto $Q H$ and by $\lambda_{m}^{k}$ the eigenvalues of $\left.\mathcal{A}\right)$.

Proof of Lemma 9. Consider the equation for the eigenvectors $\left\{\widetilde{\varphi}_{m}^{k}\right\}$ and eigenvalues $\left\{\tilde{\lambda}_{m}^{k}\right\}$ of the operator $\mathcal{A}+\mathcal{B F}=\mathcal{A}+\mathbf{b}\langle\cdot, f\rangle_{1}$, i.e.

$$
\left(\mathcal{A}-\widetilde{\lambda}_{m}^{k} I+\mathbf{b}\langle\cdot, f\rangle_{1}\right) \widetilde{\varphi}_{m}^{k}=0
$$

Now we apply the resolvent $R_{\widetilde{\lambda}_{m}^{k}}(\mathcal{A})$ to the both sides of $(47)$ to get

$$
\widetilde{\varphi}_{m}^{k}+R_{\widetilde{\lambda}_{m}^{k}}(\mathcal{A}) \mathbf{b} \cdot\left\langle\widetilde{\varphi}_{m}^{k}, f\right\rangle_{1}=0
$$


then multiply it by $f$ in the new scalar product $\langle\cdot, \cdot\rangle_{1}$ and obtain

$$
\left\langle\widetilde{\varphi}_{m}^{k}, f\right\rangle_{1}+\left\langle R_{\widetilde{\lambda}_{m}^{k}}(\mathcal{A}) \mathbf{b}, f\right\rangle_{1} \cdot\left\langle\widetilde{\varphi}_{m}^{k}, f\right\rangle_{1}=0
$$

or equivalently

$$
\left\langle R_{\widetilde{\lambda}_{m}^{k}}(\mathcal{A}) \mathbf{b}, f\right\rangle_{1}=-1
$$

Using (46), the decomposition

$$
\mathbf{b}=\sum_{j \in I} \sum_{|i| \geq N}^{\infty} \varphi_{j}^{i} \cdot\left\langle\mathbf{b}, \varphi_{j}^{i}\right\rangle_{0}+P \mathbf{b}
$$

and the explicit form of the resolvent

$$
R_{\widetilde{\lambda}_{m}^{k}}(\mathcal{A}) \mathbf{b}=\sum_{j \in I} \sum_{|i| \geq N}^{\infty} \frac{\left\langle\mathbf{b}, \varphi_{j}^{i}\right\rangle_{0}}{\lambda_{j}^{i}-\widetilde{\lambda}_{m}^{k}} \varphi_{j}^{i}+R_{\widetilde{\lambda}_{m}^{k}}(\mathcal{A}) P \mathbf{b}
$$

we can write

$$
q_{m}^{k}=\frac{\varphi_{m}^{k}}{\xi_{m}^{k}}+\frac{1}{\xi_{m}^{k}} \frac{\lambda_{m}^{k}-\widetilde{\lambda}_{m}^{k}}{\left\langle\mathbf{b}, \varphi_{m}^{k}\right\rangle_{0}}\left(\sum_{j \in I} \sum_{\substack{|i| \geq N,(i, j) \neq(k, m)}} \frac{\left\langle\mathbf{b}, \varphi_{j}^{i}\right\rangle_{0}}{\lambda_{j}^{i}-\widetilde{\lambda}_{m}^{k}} \varphi_{j}^{i}+R_{\widetilde{\lambda}_{m}^{k}}(\mathcal{A}) P \mathbf{b}\right)
$$

Using the definition of $\|\cdot\|_{1}$ by (43), we can write

$$
\left\|q_{m}^{k}-\frac{\varphi_{m}^{k}}{\xi_{m}^{k}}\right\|_{1}^{2}=\sum_{r \in I} \sum_{\substack{|i| \geq N \\(i, r) \neq(k, m)}}\left(\xi_{r}^{i}\right)^{2}\left|\left\langle q_{m}^{k}-\frac{\varphi_{m}^{k}}{\xi_{m}^{k}}, \varphi_{r}^{i}\right\rangle_{0}\right|^{2}+\left\|P\left(q_{m}^{k}-\frac{\varphi_{m}^{k}}{\xi_{m}^{k}}\right)\right\|_{0}^{2} .
$$

The second term reads (see (50))

$$
\left\|P\left(q_{m}^{k}-\frac{\varphi_{m}^{k}}{\xi_{m}^{k}}\right)\right\|_{0}^{2}=\left\|P R_{\widetilde{\lambda}_{m}^{k}}(\mathcal{A}) P \mathbf{b}\right\|_{0}^{2}=\left\|R_{\widetilde{\lambda}_{m}^{k}}(\mathcal{A}) P \mathbf{b}\right\|_{0}^{2} .
$$

Let us consider the first term in (51).

$$
\begin{aligned}
& \sum_{r \in I} \sum_{\substack{|i| \geq N \\
(i, r) \neq(k, m)}}\left(\xi_{r}^{i}\right)^{2}\left|\frac{1}{\xi_{m}^{k}} \cdot \frac{\lambda_{m}^{k}-\widetilde{\lambda}_{m}^{k}}{\left\langle\mathbf{b}, \varphi_{m}^{k}\right\rangle_{0}} \cdot \frac{\left\langle\mathbf{b}, \varphi_{r}^{i}\right\rangle_{0}}{\lambda_{r}^{i}-\widetilde{\lambda}_{m}^{k}}\right|^{2}= \\
& \quad=\left|\lambda_{m}^{k}-\widetilde{\lambda}_{m}^{k}\right|^{2} \cdot \sum_{r \in I} \sum_{\substack{i, \mid \geq N \\
(i, r) \neq(k, m)}} \frac{1}{\left|\lambda_{r}^{i}-\widetilde{\lambda}_{m}^{k}\right|^{2}} \cdot\left|\frac{\xi_{r}^{i}\left\langle\mathbf{b}, \varphi_{r}^{i}\right\rangle_{0}}{\xi_{m}^{k}\left\langle\mathbf{b}, \varphi_{m}^{k}\right\rangle_{0}}\right|^{2}
\end{aligned}
$$


We recall (cf. Theorem 2 in [18] for the neutral type system) that $\lambda_{m}^{k} \in \sigma(\mathcal{A})$ satisfies $\lambda_{m}^{k} \in L_{m}^{(k)}$ and the center of $L_{m}^{(k)}$ is $\lambda_{m}^{(k)}$. It gives that the distance between $\lambda_{m}^{(k)}$ and $\lambda_{m}^{(i)}$ is $\left|\lambda_{m}^{(k)}-\lambda_{m}^{(i)}\right|=2 \pi|k-i|$ and $\left|\lambda_{m}^{(k)}-\lambda_{r}^{(i)}\right|>2 \pi|k-i|$ for $r \neq m$. Hence, using (45) and the convergence of $\sum_{|k| \geq N, k \neq i}|k-i|^{-2}<+\infty$, we get

$$
\sum_{i \neq k,|i| \geq N} \frac{1}{\left|\lambda_{r}^{i}-\widetilde{\lambda}_{m}^{k}\right|^{2}}<+\infty .
$$

The last convergence with the property

$$
\left|\frac{\xi_{r}^{i}\left\langle\mathbf{b}, \varphi_{r}^{i}\right\rangle_{0}}{\xi_{m}^{k}\left\langle\mathbf{b}, \varphi_{m}^{k}\right\rangle_{0}}\right|=1
$$

which follows from (44), give the convergence (see (53))

$$
\sum_{r \in I} \sum_{\substack{i, i \mid \geq N \\(i, r) \neq(k, m)}} \frac{1}{\left|\lambda_{r}^{i}-\widetilde{\lambda}_{m}^{k}\right|^{2}} \cdot\left|\frac{\xi_{r}^{i}\left\langle\mathbf{b}, \varphi_{r}^{i}\right\rangle_{0}}{\xi_{m}^{k}\left\langle\mathbf{b}, \varphi_{m}^{k}\right\rangle_{0}}\right|^{2}<+\infty .
$$

Using (52)-(54), we infer from (51)

$$
\left\|q_{m}^{k}-\frac{\varphi_{m}^{k}}{\xi_{m}^{k}}\right\|_{1}^{2} \leq C \cdot\left|\lambda_{m}^{k}-\widetilde{\lambda}_{m}^{k}\right|^{2}+\left\|R_{\widetilde{\lambda}_{m}^{k}}(\mathcal{A}) P \mathbf{b}\right\|_{0}^{2},
$$

where $C$ is independent of $k, m$.

We need the following estimate which immediately follows from H1), (45) and (38):

$$
\sum_{m \in I} \sum_{|k| \geq N}\left|\lambda_{m}^{k}-\widetilde{\lambda}_{m}^{k}\right|^{2}<+\infty
$$

Now, for $R_{\widetilde{\lambda}_{m}^{k}}(\mathcal{A}) P \mathbf{b}$, we need the next estimate

$$
\sum_{m \in I} \sum_{|k| \geq N}\left\|R_{\widetilde{\lambda}_{m}^{k}}(\mathcal{A}) P \mathbf{b}\right\|_{0}^{2}<+\infty
$$

To get this, we first split the projector $P$ on a sum of projectors $P_{j}^{(r)}, j \notin I$ defined by (39):

$$
\left\|R_{\widetilde{\lambda}_{m}^{k}}(\mathcal{A}) P \mathbf{b}\right\|_{0}^{2}=\sum_{j \notin I} \sum_{|r| \geq N}\left\|R_{\widetilde{\lambda}_{m}^{k}}(\mathcal{A}) P_{j}^{(r)} \mathbf{b}\right\|_{0}^{2}
$$

Now, using formula similar to (22) (with $\widetilde{\lambda}_{m}^{k}$ instead of $\mathrm{e}^{\lambda}$ ), we deduce that 


$$
\begin{aligned}
\left\|R_{\widetilde{\lambda}_{m}^{k}}(\mathcal{A}) P_{j}^{(r)} \mathbf{b}\right\|_{0}^{2} & \leq\left\|P_{j}^{(r)} \mathbf{b}\right\|_{0}^{2} \cdot\left(\operatorname{dist}\left(\widetilde{\lambda}_{m}^{k}, L_{j}^{(r)}\right)\right)^{-2} \\
& \leq\left\|P_{j}^{(r)} \mathbf{b}\right\|_{0}^{2} \cdot\left(2 \pi r_{0} \max \{|k-r|, 1\}\right)^{-2}
\end{aligned}
$$

The last estimate holds due to the location of the circles $L_{j}^{(r)}$ of radius $r_{0}$ and centered at $\lambda_{j}^{(r)}$. Since for any fixed $n$ the series $\sum_{k}\left(2 \pi r_{0} \max \{|k-r|, 1\}\right)^{-2}$ absolutely converges and $\sum_{k \notin I,|r| \geq N}\left\|P_{k}^{(r)} \mathbf{b}\right\|_{0}^{2}<+\infty$ (since $\left.\mathbf{b} \in H\right)$, one has

$$
\begin{gathered}
\sum_{m \in I} \sum_{|k| \geq N}\left\|R_{\widetilde{\lambda}_{m}^{k}}(\mathcal{A}) P \mathbf{b}\right\|_{0}^{2} \leq \sum_{|k| \geq N} \sum_{j \notin I,|r| \geq N}\left\|P_{j}^{(r)} \mathbf{b}\right\|_{0}^{2} \cdot\left(2 \pi r_{0} \max \{|k-r|, 1\}\right)^{-2} \\
\leq \sum_{j \notin I,|r| \geq N}\left\|P_{j}^{(r)} \mathbf{b}\right\|_{0}^{2} \cdot\left\{\sum_{|k| \geq N}\left(2 \pi r_{0} \max \{|k-r|, 1\}\right)^{-2}\right\}<+\infty .
\end{gathered}
$$

So we get (57).

We notice that $\left\|\varphi_{m}^{k}\right\|_{1}=\xi_{m}^{k}$, so the vectors $\left\{\frac{\varphi_{m}^{k}}{\xi_{m}^{k}}\right\}$ form an orthonormal basis (in the norm $\|\cdot\|_{1}$ ) in the closure of their linear span. Using (56),(57) we deduce from (55) that

$$
\sum_{m \in I} \sum_{|k| \geq N}\left\|q_{m}^{k}-\frac{\varphi_{m}^{k}}{\xi_{m}^{k}}\right\|_{1}^{2}<+\infty
$$

Hence there exists $N_{2} \in \mathbb{N}$, such that

$$
\sum_{m \in I} \sum_{|k| \geq N_{2}}\left\|q_{m}^{k}-\frac{\varphi_{m}^{k}}{\xi_{m}^{k}}\right\|_{1}^{2}<1
$$

Then the sequence $\left\{q_{m}^{k}\right\}$ is quadratically close to the orthonormal basis $\left\{\frac{\varphi_{m}^{k}}{\xi_{m}^{k}}\right\}$ and Theorem 5.2 [5] (see also Theorem 2.20 and Corollary 2.22 in [8]) gives that $\left\{q_{m}^{k}\right\}_{m \in I,|k| \geq N_{2}}$ form a Riesz basis of the closure (in $\|\cdot\|_{1}$ ) of their linear $\operatorname{span} Q H=\operatorname{Clspan}\left\{q_{m}^{k}: m \in I,|k| \geq N_{2}\right\}$, where $Q$ is the orthogonal, in $\|\cdot\|_{1}$, projector onto $Q H$. This completes the proof of Lemma 9.

Using (48),(46), we consider

$$
\left\langle q_{m}^{k}, f\right\rangle_{1}=\frac{1}{\xi_{m}^{k}} \cdot \frac{\lambda_{m}^{k}-\widetilde{\lambda}_{m}^{k}}{\left\langle b, \varphi_{m}^{k}\right\rangle_{0}} \cdot\left\langle R_{\widetilde{\lambda}_{m}^{k}}(\mathcal{A}) \mathbf{b}, f\right\rangle_{1}=-\frac{1}{\xi_{m}^{k}} \cdot \frac{\lambda_{m}^{k}-\widetilde{\lambda}_{m}^{k}}{\left\langle\mathbf{b}, \varphi_{m}^{k}\right\rangle_{0}} .
$$

Taking into account assumption (44) and (56), one has

$$
\sum_{m \in I} \sum_{|k| \geq N_{2}}\left|\left\langle q_{m}^{k}, f\right\rangle_{1}\right|^{2}=\sum_{m \in I} \sum_{|k| \geq N_{2}}\left|\lambda_{m}^{k}-\tilde{\lambda}_{m}^{k}\right|^{2}<+\infty
$$


The Riesz basis property of $\left\{q_{m}^{k}\right\}_{m \in I,|k| \geq N_{2}}$ and (61) give the existence and uniqueness of $\tilde{f} \in Q H$ such that $\|\tilde{f}\|_{1}$ is finite and (see (60))

$$
\left\langle q_{m}^{k}, \widetilde{f}\right\rangle_{1}=-\frac{1}{\xi_{m}^{k}} \cdot \frac{\lambda_{m}^{k}-\widetilde{\lambda}_{m}^{k}}{\left\langle\mathbf{b}, \varphi_{m}^{k}\right\rangle_{0}} .
$$

Finally, there exists a unique $f \in H$ such that $\|f\|_{1}$ is finite, $Q f=\widetilde{f}$ and $(I d-Q) f=0$.

By construction, the feedback control $u=\mathcal{F} x$ defined by $\mathcal{F}: \mathcal{D}(\mathcal{A}) \rightarrow \mathbb{C}$, and $\mathcal{F} x \stackrel{\text { def }}{=}\langle x, f\rangle_{1}$ gives the eigenvalues $\widetilde{\lambda}_{m}^{k}$ for the operator $\mathcal{A}+\mathcal{B F}$ and, as a result, proves property 1 ) of Theorem 8 . The property 2) of Theorem 8 follows immediately from the definition of the new norm (43) and properties (44), (41). This completes the proof of Theorem 8.

\section{Stabilization of the neutral type system}

Now we can prove the main result of the paper, namely Theorem 1 . To this end we give another formulation of this result.

Theorem 10 Let us denote by $b_{1}, \ldots, b_{p} \in \mathbb{C}^{n}$ the columns of the matrix $B$. Assume the following four conditions are satisfied.

1) All the eigenvalues of the matrix $A_{-1}$ satisfy $|\mu| \leq 1$.

2) All the eigenvalues $\mu_{j} \in \sigma\left(A_{-1}\right) \cap\{z:|z|=1\}$ are simple (we denote their index $j \in I)$.

3) $\sum_{i=1}^{p}\left|\left\langle b_{i}, y_{m}^{k}\right\rangle_{\mathbb{C}^{n}}\right| \neq 0$ for all the vectors $y_{m}^{k}$ satisfying $y_{m}^{k} \in \operatorname{Ker} \Delta_{\mathcal{A}}^{*}(\lambda)$ for roots $\lambda$ of the equation det $\Delta_{\mathcal{A}}^{*}(\lambda)=0$, such that $\operatorname{Re} \lambda \geq 0$. Here $m \in I$ and $\Delta_{\mathcal{A}}^{*}(\lambda)$ is defined in (28).

4) $\sum_{i=1}^{p}\left|\left\langle b_{i}, y_{m}\right\rangle_{\mathbb{C}^{n}}\right| \neq 0$ for all the eigenvectors $y_{m}$ of the matrix $A_{-1}^{*}$, corresponding to eigenvalues $\bar{\mu}_{m},\left|\mu_{m}\right|=1$.

Then there exists a regular control $u=\mathcal{F} x$ of the form (10), which stabilizes the system (2) (and (6)), i.e. $\mathcal{D}(\mathcal{A})=\mathcal{D}(\mathcal{A}+\mathcal{B F})$ and $\mathrm{e}^{(\mathcal{A}+\mathcal{B} F) t} x_{0} \rightarrow 0$ as $t \rightarrow \infty$ for all $x_{0} \in M_{2}$.

Remark 11 It is easy to see that conditions 1) and 2) of Theorem 1 and Theorem 10 coincide. Conditions 3) and 4) of the same theorems are equivalent.

Proof. For simplicity of reading let us give a brief sketch of the proof. The details are given in the following section. 
The proof is given in two steps. The first step is devoted to the assignment of an infinite part of the spectrum. Namely, we show that there exists $N$, big enough, such all the eigenvalues $\lambda_{m}^{k}$ of the operator $\mathcal{A}$ such that $|k|>N$ corresponding to the eigenvalue $\mu_{m}$ of the matrix $A_{-1}$ and such that $\left|\mu_{m}\right|=1$ may be moved arbitrarily in the circle $L_{m}^{(k)}$. This is based on the application of the Theorem 8. As this result is valid for a single control, we first show that one can reduce this multivariable problem to a single input problem. The integer $N$ is also chosen such that the other circles $L_{m}^{(k)}$ (for which $\left|\mu_{m}\right| \neq 1$ ) are in the open left half plane (recall that the radii of that circles verify: $r^{k} \rightarrow 0$ as $\left.k \rightarrow \infty\right)$. In other words we move only "bad" eigenvalues close to the imaginary axis. This "infinite part" of the spectrum may characterized also as $\{\lambda \in \sigma(\mathcal{A}):|\operatorname{Im} \lambda|>K\}$ for some $K>0$.

The second step is concerned with the assignment of the finite part of the spectrum, i.e. the part $\{\lambda \in \sigma(\mathcal{A}):|\operatorname{Im} \lambda| \leq K\}$. This is a finite dimensional problem of stabilizability: all the controllable eigenvalues may be moved arbitrarily by a regular feedback.

Finally, it is shown, that the uncontrollable eigenvalues, which are, by hypothesis "asymptoticaly" stable, i.e. in the open left half plane are not affected by the stabilizing feedback.

\section{Proof of the main result}

Here we give the detailed proof of Theorem 10. This proof is divided in two parts. The main contribution here is the assignment of the controlled eigenvalues near the imaginary axis.

\subsection{Assignment of an infinite family of eigenvalues}

This part of the proof is based on Theorem 8. However, this Theorem is proved for the case of a scalar control $u$. Let us first reduce a part of the problem to this single input case. Namely, we prove that under the conditions of the Theorem 10, for the abstract system (6) there exists a vector $\mathbf{b}=(b, 0) \in H=$ $M_{2}$ such that the conditions of Theorem 8 are satisfied.

First we prove the following Lemmas.

Lemma 12 Assume that the condition 2) of Theorem 10 is satisfied. Consider the eigenvectors $y_{m},\left(\left\|y_{m}\right\|=1\right)$ of $A_{-1}^{*}$, for $m \in I$, where

$$
I=\left\{\bar{\mu}_{m} \in \sigma\left(A_{-1}^{*}\right):\left|\mu_{m}\right|=1\right\} .
$$


Assume that for some vector $b \in \operatorname{Im} B$ and for all $m \in I$ one has $\left\langle b, y_{m}\right\rangle_{\mathbb{C}^{n}} \neq 0$. Then

$$
\left|\left\langle b, y_{m}^{k}\right\rangle_{\mathbb{C}^{n}}\right| \sim C_{m} \cdot(|k|+1)^{-1} \quad \text { as } \quad k \rightarrow \infty, \quad m \in I, \quad C_{m} \in \mathbb{R} .
$$

Proof. Consider an eigenvector of $\mathcal{A}^{*}$, which expression is given in (27), such that $\left\|\left(\begin{array}{c}y_{m}^{k} \\ \psi_{m}^{k}(\cdot)\end{array}\right)\right\|_{M_{2}}=1$ and let us show that

$$
\left\|y_{m}^{k}\right\|_{\mathbb{C}^{n}} \sim C_{m} \cdot(|k|+1)^{-1} \quad \text { as } \quad k \rightarrow \infty, \quad m \in I, \quad C_{m} \in \mathbb{C} .
$$

Using the expression (27) for the eigenvectors of the operator $\mathcal{A}^{*}$, we get for $\lambda=\lambda_{m}^{k}$ that $1=\left\|\left(\begin{array}{c}y_{m}^{k} \\ \psi_{j}^{k}(\cdot)\end{array}\right)\right\|_{M_{2}}^{2}=\left\|y_{m}^{k}\right\|_{\mathbb{C}^{n}}^{2} \cdot k_{1}(\lambda)$, where $k_{1}(\lambda)$ is given by the expression

$$
\begin{array}{r}
1+\int_{-1}^{0}\left|\lambda \mathrm{e}^{-\lambda \theta}-A_{2}^{*}(\theta)+\mathrm{e}^{-\lambda \theta} \int_{0}^{\theta} \mathrm{e}^{\lambda s} A_{3}^{*}(s) \mathrm{d} s+\lambda \mathrm{e}^{-\lambda \theta} \int_{0}^{\theta} \mathrm{e}^{\lambda s} A_{2}^{*}(s) \mathrm{d} s\right|^{2} \mathrm{~d} \theta= \\
1+|\lambda|^{2} \int_{-1}^{0}\left|\mathrm{e}^{-\lambda \theta}\left[I+\int_{0}^{\theta} \mathrm{e}^{\lambda s} A_{2}^{*}(\theta) \mathrm{d} s\right]+\lambda^{-1}\left[-A_{2}^{*}(\theta)+\mathrm{e}^{-\lambda \theta} \int_{0}^{\theta} \mathrm{e}^{\lambda s} A_{3}^{*}(s) \mathrm{d} s\right]\right|^{2} \mathrm{~d} \theta .
\end{array}
$$

Since $\int_{-1}^{0}\left|\mathrm{e}^{-\lambda_{m}^{k} \theta}\right|^{2} \mathrm{~d} \theta \rightarrow 1$, as $i \rightarrow \infty$ (see Theorem 4 on the location of the spectrum), and all the other terms tend to zero, we get $1=\left\|\left(\begin{array}{c}y_{m}^{k} \\ \psi_{m}^{k}(\cdot)\end{array}\right)\right\|_{M_{2}}^{2} \sim$ $\left\|y_{m}^{k}\right\|_{\mathbb{C}^{n}}^{2} \cdot\left(1+|\lambda|^{2}\right)$.

Now let us consider an eigenvector $y_{m},\left(\left\|y_{m}\right\|=1\right)$ of $A_{-1}^{*}$, corresponding to an eigenvalue $\bar{\mu}_{m},\left|\mu_{m}\right|=1$ (all these eigenvalues are simple). We are also interested in the unit eigenvector $y_{m}^{k} \cdot\left\|y_{m}^{k}\right\|^{-1}$ of $\Delta_{\mathcal{A}}^{*}\left(\lambda_{m}^{k}\right)+\lambda_{m}^{k} I$ (see (28) for the definition of $\left.\Delta_{\mathcal{A}}^{*}(\lambda)\right)$, i.e. $y_{m}^{k} \in \operatorname{Ker} \Delta^{*}\left(\lambda_{m}^{k}\right)$. The eigenvalues are the roots of equation $\operatorname{det} \Delta^{*}(\lambda)=0$.

One can easily check that $\left\|y_{m}-y_{m}^{k} \cdot\right\| y_{m}^{k}\left\|^{-1}\right\|_{\mathbb{C}^{n}} \rightarrow 0$ as $k \rightarrow \infty$. This is due to the continuous dependence of the coefficients of the $n$-dimensional matrix $\Delta^{*}(\lambda)$ on the parameter $\lambda$ (see (28)). Hence for $k \rightarrow \infty$ we deduce (we remind that by assumption $\left.\left\langle b, y_{m}\right\rangle_{\mathbb{C}^{n}} \neq 0\right)$

$$
\lim _{k \rightarrow \infty}\left|\frac{\left\langle b, y_{m}-y_{m}^{k} \cdot\left\|y_{m}^{k}\right\|^{-1}\right\rangle_{\mathbb{C}^{n}}}{\left\langle b, y_{m}\right\rangle_{\mathbb{C}^{n}}}\right| \leq \lim _{k \rightarrow \infty} \frac{\|b\| \cdot\left\|y_{m}-y_{m}^{k} \cdot\right\| y_{m}^{k}\left\|^{-1}\right\|_{\mathbb{C}^{n}}}{\left|\left\langle b, y_{m}\right\rangle_{\mathbb{C}^{n}}\right|}=0
$$

Hence

$$
\frac{\left|\left\langle b, y_{m}^{k} \cdot\left\|y_{m}^{k}\right\|^{-1}\right\rangle_{\mathbb{C}^{n}}\right|}{\left|\left\langle b, y_{m}\right\rangle_{\mathbb{C}^{n}}\right|}=\left|\frac{\left\langle b, y_{m}-y_{m}^{k} \cdot\left\|y_{m}^{k}\right\|^{-1}\right\rangle_{\mathbb{C}^{n}}}{\left\langle b, y_{m}\right\rangle_{\mathbb{C}^{n}}}-1\right| \rightarrow 1 \text { as } i \rightarrow \infty .
$$


Using this, one has

$$
\begin{aligned}
\lim _{k \rightarrow \infty} \frac{\left|\left\langle b, y_{m}^{k} \cdot\left\|y_{m}^{k}\right\|^{-1}\right\rangle_{\mathbb{C}^{n}}\right|}{\left|\left\langle b, y_{m}\right\rangle_{\mathbb{C}^{n}}\right|} & =\lim _{k \rightarrow \infty} \frac{\|b\| \cdot 1 \cdot\left|\cos \left(\widehat{b, y_{m}^{k}}\right)\right|}{\|b\| \cdot 1 \cdot\left|\cos \left(\widehat{b, y_{m}}\right)\right|} \\
& =\lim _{k \rightarrow \infty} \frac{\left|\cos \left(\widehat{b, y_{m}^{k}}\right)\right|}{\left|\cos \left(\widehat{b, y_{m}}\right)\right|}=1
\end{aligned}
$$

The last property implies

$$
\left|\left\langle b, y_{m}^{k}\right\rangle_{\mathbb{C}^{n}}\right|=\|b\| \cdot\left\|y_{m}^{k}\right\| \cdot\left|\cos \left(\widehat{b, y_{m}^{k}}\right)\right| \sim\|b\| \cdot\left\|y_{m}^{k}\right\| \cdot\left|\cos \left(\widehat{b, y_{m}}\right)\right|, \quad k \rightarrow \infty .
$$

Estimate (64) gives (63) and completes the proof of Lemma 12.

Lemma 13 Assume that all the conditions of Theorem 10 are satisfied. Then there exists a vector $b \in \operatorname{Im} B$ say $b=c_{1} b_{1}+\ldots+c_{p} b_{p}$, which satisfies the conditions:

1) $\left\langle b, y_{m}\right\rangle_{\mathbb{C}^{n}} \neq 0$ for all the eigenvectors $y_{m}$ of the matrix $A_{-1}^{*}$, corresponding to eigenvalues $\bar{\mu}_{m},\left|\mu_{m}\right|=1$;

2) $\left\langle b, y_{m}^{k}\right\rangle_{\mathbb{C}^{n}} \neq 0$ for vectors $y_{m}^{k}$ satisfying $y_{m}^{k} \in \operatorname{Ker} \Delta^{*}(\lambda)$ for roots $\lambda$ of the equation $\operatorname{det} \Delta^{*}(\lambda)=0$, such that $\operatorname{Re} \lambda \geq 0$ and for all $k$ such that $|k| \geq N_{3}$ for some integer $N_{3}$.

Proof. It is enough to prove that there exists $b$ such that 1 ) is satisfied. The statement 2) is a consequence of Lemma 12.

Let $y_{m}, m \in I$ be eigenvectors of the matrix $A_{-1}^{*}$, corresponding to eigenvalues $\bar{\mu}_{m}$, such that $\left|\mu_{m}\right|=1$. Consider the subspaces

$$
\mathcal{L}_{m}=\left\{y:\left\langle y, y_{m}\right\rangle_{\mathbb{C}^{n}}=0\right\}, \quad m \in I .
$$

They are of dimension $n-1$. Let us denote by $\mathcal{M}_{m}$ the subspaces $\mathcal{M}_{m}=$ $\mathcal{L}_{m} \cap \operatorname{Im} B$. We have by Assumption 3) of Theorem $10 \operatorname{dim} \mathcal{L}_{j}<p$. Indeed, if $\operatorname{dim} \mathcal{L}_{m}=p=\operatorname{dim}(\operatorname{Im} B)$, then $B^{*} y_{m}=0$ and the condition 3$)$ is not satisfied. This gives that $\mathcal{L}_{m}, m \in I$ are nowhere dense in $\operatorname{Im} B$. Then by the Baire theorem

$$
\bigcup_{m \in I} \mathcal{M}_{m} \neq \operatorname{Im} B
$$

This means that there exists $b \in \operatorname{Im} B$ such that $b \notin \mathcal{L}_{m}$ fo all $m \in I$, i.e. $\left\langle b, y_{m}\right\rangle \neq 0, m \in I$. It implies that there does not exist an eigenvector $x$ corresponding to an eigenvalue $\bar{\mu},|\mu|=1$ of the matrix $A_{-1}$ such that $\langle x, b\rangle=0$. Indeed, if such a vector exists then it is colinear to some vector $x_{m}$, 
because of the simplicity of the spectrum and then $\left\langle y_{m}, b\right\rangle=0$, which is in contradiction the construction of $b$.

Let us now give the proof of the assignement of an infinite part of the spectrum of $\mathcal{A}$.

We show first that the operator $\mathcal{A}$, defined in (6), and corresponding to the neutral type system (2), satisfies assumptions H1) and H2) of Section 3, so we can apply Theorem 8 after a reduction to a single control case (see below).

We naturally put $H \stackrel{\text { def }}{=} M_{2}=\mathbb{C}^{n} \times L_{2}\left(-1,0 ; \mathbb{C}^{n}\right)$. Property H1) is a consequence of Theorem 4, while property $\mathrm{H} 2$ ) follows from the next theorem (see theorems 15 and 16 from [18] and also [16] for more details and proof).

Theorem 14 [18] There exists a sequence of invariant for $\mathcal{A}$ finite-dimensional subspaces which constitute a Riesz basis in $M_{2}$.

More precisely, there exists $N_{0}$ large enough, such that for any $N \geq N_{0}$, these subspaces are $\left\{V_{m}^{(k)},|k| \geq N, m=1, . ., \ell\right\}$ and $W_{N}$, where the last one is the $2(N+1) n$-dimensional subspace spanned by all eigen- and rootvectors, corresponding to all eigenvalues of $\mathcal{A}$, which are outside of all circles $L_{m}^{(k)},|k| \geq N, m=1, . ., \ell$.

The conditions of the Theorem 10 allow to apply Lemma 13. Then we have a vector $\mathbf{b}=(b, 0)$ such that all the conditions of the abstract result on stabilization (Theorem 8) are satisfied. Let us take now $N$ sufficiently large, greater than $N_{0}, N_{1}, N_{2}$ and $N_{3}$ (see Theorem 14, Lemmas 9, 12, 13), and such that the circles $L_{m}^{(k)},|k|>N$ for $m \notin I$ are in the open left half plane (recall that $I$ is the set such that the eigenvalues $\mu_{m}, m \in I$ of the matrix $A_{-1}$ verify $\left.\left|\mu_{m}\right|=1\right)$. We then choose the scalar $\widetilde{\lambda}_{m}^{k}$ for $|k|>N$, in the left half plane. Let the corresponding feedback be noted by $\mathcal{F}_{1}$. We can observe that

$$
\mathbf{b}=\left(\begin{array}{l}
b \\
0
\end{array}\right)=\left(\begin{array}{c}
c_{1} b_{1}+\ldots+c_{p} b_{p} \\
0
\end{array}\right)=\left(\begin{array}{c}
B \\
0
\end{array}\right) c=\mathcal{B} c, \quad c=\left(\begin{array}{c}
c_{1} \\
\vdots \\
c_{p}
\end{array}\right)
$$

The operator $\mathcal{F}_{1}$ may be written as (see the proof of Theorem 8): $\mathcal{F}_{1} x=$ $\langle x, f\rangle_{1}$. Consider now the operator $\mathcal{F}_{2}$ defined by

$$
\mathcal{F}_{2}: M_{2} \rightarrow \mathbb{C}^{p}, \quad \mathcal{F}_{2} x=c\langle x, f\rangle_{1}
$$

This operator moves the eigenvalues $\lambda_{m}^{k}$, near the imaginary axis, to the eigenvalues $\widetilde{\lambda}_{m}^{k}$, near the imaginary axis also, into the circles $L_{m}^{(k)}$, but in the left half plane, the integer $m$ being in $I$. This end the proof for the assignment of an infinite part of the spectrum. 


\subsection{Assignment of a finite family of eigenvalues}

After the first step described above we obtain a new neutral type systems with a finite number of unstable eigenvalues. The above described procedure can be realized in such a way the new eigenvalues do not coincide with some "old" stable eigenvalues. We can then, one more time apply Theorem 14. This gives that the space $M_{2}$ is decomposed in a sum of two $\mathcal{A}+\mathcal{B} \mathcal{F}_{1}$-invariant subspaces $H_{1}$, corresponding to the infinite part of the spectrum, and $H_{2}$, corresponding to the finite part of the spectrum, i.e. for all $x \in M_{2}$, we have $x=x_{1}+x_{2}, x_{i} \in H_{i}, i=1,2$. Let us note by $\mathcal{A}_{11}, \mathcal{A}_{22}, \mathcal{B}_{1}$ and $\mathcal{B}_{2}$ the corresponding restrictions of the operators $\mathcal{A}+\mathcal{B} \mathcal{F}_{1}$, and $\mathcal{B}$ :

$$
\mathcal{A}+\mathcal{B F}_{1}=\left(\begin{array}{cc}
A_{11} & 0 \\
0 & A_{22}
\end{array}\right), \quad \mathcal{B}=\left(\begin{array}{c}
\mathcal{B}_{1} \\
\mathcal{B}_{2}
\end{array}\right)
$$

By the assumptions of Theorem 10 we have that the finite dimensional system $\left(\mathcal{A}_{22}, \mathcal{B}_{2}\right)$ is stabilizable. That is, there exists a feedback $u=\mathcal{F}_{22} x_{2}$ wich stabilize the system $\left(\mathcal{A}_{22}, \mathcal{B}_{2}\right)$. Then the feedback

$$
u=\mathcal{F} x=\mathcal{F}_{1} x+\mathcal{F}_{2} x=\mathcal{F}_{1} x+\left(\begin{array}{ll}
0 & F_{22}
\end{array}\right)\left(\begin{array}{c}
x_{1} \\
x_{2}
\end{array}\right)
$$

transform the original system into a system where all the conditions of asymptotic stability are verified [18, Theorem 22].

Let us, finally, observe that the different feedbacks do not affect the uncontrollable stable eigenvalues. Indeed, suppose that $\lambda_{0}$ be a stable uncontrollable eigenvalue, i.e. such that $\operatorname{Re} \lambda_{0}<0$ and the corresponding eigenvector $x_{0}$ verify $\mathcal{B}^{*} x_{0}=0$. Then $(\mathcal{A}+\mathcal{B} \mathcal{F})^{*} x_{0}=\mathcal{A}^{*} x_{0}+\mathcal{F}^{*} \mathcal{B}^{*} x_{0}=\mathcal{A}^{*} x_{0}=\lambda_{0} x_{0}$. This conclude the proof of Theorem 10 .

\section{Conclusion and discussion.}

Analysis carried out in this work was focused on the case when the part of the spectrum $\sigma_{1}=\sigma\left(A_{-1}\right) \cap\{z:|z|=1\}$ consists of simple eigenvalues and $\sigma\left(A_{-1}\right) \subset\{z:|z| \leq 1\}$. In this case we proved that the system (2) can be stabilized by a set of regular controls of the form (10). This set is infinite since any sequence $\left\{\widetilde{\lambda}_{m}^{n}\right\}$, satisfying (45), and such that $\operatorname{Re} \widetilde{\lambda}_{m}^{n}<0$, gives a stabilizing control.

On the other hand, in the case when $\sigma_{1}$ has at least one eigenvalue of $A_{-1}$ with a nontrivial Jordan chain, the system (2) can not be stabilized by a control of the form (10). The same if $\sigma\left(A_{-1}\right) \not \subset\{z:|z| \leq 1\}$. This follows from the fact 
that any control of the form (10) leaves the system in the same form (compare (1) and (12)), hence Theorem 24 from [18] says that the system is unstable.

The remaining case when there are no Jordan blocks, corresponding to eigenvalues in $\sigma_{1}$, but there exists $\mu \in \sigma_{1}$ whose eigenspace is at least two-dimensional (see part p3) of Theorem 22 from [18]), is much more complicated as clearly showed in paragraph 3.2.2 of [18]. This case needs further investigations.

\section{References}

[1] Bellman R., Cooke K. L. Differential-difference equations, in "Mathematics in Science and Engineering". vol. 6. New York-London: Academic Press, 1963.

[2] Brumley W.E., On the asymptotic behavior of solutions of differential-difference equations of neutral type, J. Differential Equations 7(1970), 175-188.

[3] Burns, J. A., Herdman, T.L., Stech, H. W, Linear functional-differential equations as semigroups on product spaces. SIAM J. Math. Anal., 14(1983), No. $1,98-116$.

[4] Diekmann O., van Gils S. A., Verduyn Lunel S. M., Walther H.-O., Delay equations. Functional, complex, and nonlinear analysis, Springer-Verlag, New York, 1995.

[5] Gohberg I. C., Krein M. G. Introduction to the theory of linear non-selfadjoint operators (English) Translations of Mathematical Monographs. 18. Providence, RI: AMS. XV, 378 p. (1969).

[6] Hale J., Verduyn Lunel S. M, "Theory of functional differential equations", Springer-Verlag, New York, 1993.

[7] Hale, J. K., Verduyn Lunel, S. M. Strong stabilization of neutral functional differential equations. IMA Journal of Mathematical Control and Information, 19(2002), No 1/2, 5-23.

[8] Kato T., Perturbation theory for linear operators, Springer Verlag, 1966.

[9] Ito K. and Tarn T. J., A linear quadratic optimal control for neutral systems, Nonlinear Analysis, Theory, Methods \& Applications, 9(1985), No 7, 699-727.

[10] O'Connor D. A. and Tarn T. J., On stabilization by state feedback for neutral differential equations, IEEE Transactions on Automatic Control, AC-28(1983), No 5, 615-618

[11] Pandolfi L., Stabilization of neutral functional differential equations, $J$. Optimization Theory and Appl., 20(1976), No. 2, 191-204.

[12] Oostveen J. C., Strongly stabilizable infinite-dimensional systems. PhD Thesis, University of Groningen, the Netherlands, 1999. 
[13] Rabah R., Sklyar G. M., Strong stabilizability for a class of linear time delay systems of neutral type. Matem. Fizika, Analiz, Geometria, 11(2004), No 3, 314330 .

[14] Rabah R., Sklyar G. M., On a class of strongly stabilizable systems of neutral type. Applied Mathematical Letters, 18(2005), No 4, 463-469.

[15] Rabah R., Sklyar G. M. The analysis of exact controllability of neutral type systems by the moment problem, approach. SIAM J. Control and Optimization, 46(2007), No 6, pp. 2148-2181.

[16] Rabah R., Sklyar G. M., Rezounenko A. V., Generalized Riesz basis property in the analysis of neutral type systems. C. R. Math. Acad. Sci. Paris, 337(2003), No. 1, 19-24.

[17] Rabah R., Sklyar G. M., Rezounenko A.V., On strong stability and stabilizability of systems of neutral type. In "Advances in time-delay systems", Lecture Notes in Comp. Sci. Engin., vol. 38, 257-268, Springer, 2004.

[18] Rabah R., Sklyar G. M., Rezounenko A. V., Stability analysis of neutral type systems in Hilbert space. J. Differential Equat., 214(2005), Issue 2, 391-428.

[19] Rabah R., Sklyar G. M., Rezounenko A.V., On strong regular stabilizability for linear neutral type systems. 14th Mediterranean Conference on Control and Automation, Ancona (Italia), June 28-30, 2006.

[20] Sklyar G. M., Rezounenko A.V., A theorem on the strong asymptotic stability and determination of stabilizing controls, C.R. Acad. Sci. Paris, Ser.I, 333(2001), No 8, 807-812.

[21] Sklyar G. M., Rezounenko A. V., Strong asymptotic stability and constructing of stabilizing controls. Matem. Fizika, Analiz, Geometria, 10(2003), No. 4, 569582 .

[22] Verduyn Lunel S. M., Yakubovich D. V., A functional model approach to linear neutral functional differential equations. Integral Equa. Oper. Theory, 27(1997), 347-378.

[23] Wonham W. M. Linear multivariable control. A geometric approach. Third edition. Springer-Verlag, New York, 1985. 